\title{
Implantation of olfactory ensheathing cells promotes neuroplasticity in murine models of stroke
}

\author{
Woei-Cherng Shyu, ${ }^{1,2}$ Demeral David Liu, ${ }^{3}$ Shinn-Zong Lin, ${ }^{1,2}$ Wen-Wen Li, ${ }^{4}$ Ching-Yuan Su, ${ }^{5}$ \\ Ying-Chen Chang, ${ }^{1,2}$ Hsiao-Jung Wang, ${ }^{1,2}$ Hsing-Won Wang, ${ }^{6}$ Chang-Hai Tsai, ${ }^{7}$ and Hung $\mathrm{Li}^{4,5}$ \\ ${ }^{1}$ Center for Neuropsychiatry, China Medical University Hospital, Taichung, Republic of China. ${ }^{2}$ Buddhist Tzu-Chi General Hospital, Hualien, Republic of China. \\ ${ }^{3}$ Department of Dentistry, China Medical University Hospital, Taichung, Republic of China. ${ }^{4}$ nnstitute of Biochemistry and Molecular Biology, \\ National Yang-Ming University, Taipei, Republic of China. Institute of Molecular Biology, Academia Sinica, Taipei, Republic of China. ${ }^{6}$ Department of ENT, \\ Tri-Service General Hospital, National Defense Medical Center, Taipei, Republic of China. ${ }^{7}$ Department of Medical Research, \\ China Medical University Hospital, Taichung, Republic of China.
}

\begin{abstract}
Murine olfactory ensheathing cells (OECs) promote central nervous system axonal regeneration in models of spinal cord injury. We investigated whether OECs could induce a neuroplastic effect to improve the neurological dysfunction caused by hypoxic/ischemic stress. In this study, human OECs/olfactory nerve fibroblasts (hOECs/ONFs) specifically secreted trophic factors including stromal cell-derived factor-1 $\alpha$ (SDF-1 $\alpha$ ). Rats with intracerebral hOEC/ONF implantation showed more improvement on behavioral measures of neurological deficit following stroke than control rats. $\left[{ }^{18} \mathrm{~F}\right]$ fluoro-2-deoxyglucose PET (FDG-PET) showed increased glucose metabolic activity in the hOEC/ONF-treated group compared with controls. In mice, transplanted hOECs/ONFs and endogenous homing stem cells including intrinsic neural progenitor cells and bone marrow stem cells colocalized with specific neural and vascular markers, indicating stem cell fusion. Both hOECs/ ONFs and endogenous homing stem cells enhanced neuroplasticity in the rat and mouse ischemic brain. Upregulation of SDF-1 $\alpha$ and CXCR4 in hOECs/ONFs promoted neurite outgrowth of cocultured primary cortical neurons under oxygen glucose deprivation conditions and in stroke animals through upregulation of cellular prion protein $\left(\mathrm{PrP}^{\mathrm{C}}\right)$ expression. Therefore, the upregulation of $\mathrm{SDF}-1 \alpha$ and the enhancement of CXCR4 and $\mathrm{Pr}^{\mathrm{C}}$ interaction induced by hOEC/ONF implantation mediated neuroplastic signals in response to hypoxia and ischemia.
\end{abstract}

\section{Introduction}

The olfactory mucosa $(\mathrm{OM})$ contains unmyelinated olfactory nerves with olfactory ensheathing cells (OECs) and sheet-like processes of olfactory nerve fibroblasts (ONFs) $(1,2)$. The OECs guide growing axons from the neurons of the nasal cavity $\mathrm{OM}$ to the olfactory bulb to form synapses in the brain. Owing to their strong ability to guide axon outgrowth, OECs have been found to assist neuroregeneration in animal models of spinal cord injury and Parkinson disease $(3,4)$. Furthermore, OECs are pluripotent cells that can show Schwann cell-like properties (5). When transplanted into the demyelinated spinal cord, OECs can repair the defective myelin and restore conductance in remyelinated axons (6-8). Although much is known about OECs, few studies have focused on the characteristics of ONFs, which are flat and compact cells forming multiple layers with thin processes encircling OECs (2).

Neurotrophic factors support neuronal survival and axonal outgrowth (9). Many trophic factors secreted from human OECs/

Nonstandard abbreviations used: BDNF, brain-derived neurotrophic factor; BMSC, bone marrow stem cell; FDG-PET, $\left[{ }^{18} \mathrm{~F}\right]$ fluoro-2-deoxyglucose PET; FN, fibronectin; GDNF, glial cell line-derived neurotrophic factor; GFAP, glial fibrillary acidic protein; hOEC, human OEC; IHC, immunohistochemistry; INPC, intrinsic neural progenitor cell; MAP-2, microtubule associated protein-2; MCA, middle cerebral artery; OE, olfactory epithelium; OEC, olfactory ensheathing cell; OGD, oxygen glucose deprivation; ONF, olfactory nerve fibroblast; $\mathrm{p} 75$, low-affinity nerve growth factor receptor; $\mathrm{PCC}$, primary cortical culture; $\mathrm{PrP}^{\mathrm{C}}$, cellular prion protein; $\mathrm{SDF}-1 \alpha$, stromal cell-derived factor- $1 \alpha$.

Conflict of interest: The authors have declared that no conflict of interest exists. Citation for this article: J. Clin. Invest. 118:2482-2495 (2008). doi:10.1172/JCI34363.
ONFs (hOECs/ONFs), such as brain-derived neurotrophic factor (BDNF), VEGF, and glial cell line-derived neurotrophic factor (GDNF), have been extensively studied to discover any potential they may have in promoting cerebral plasticity and enhancing functional recovery in animal stroke models (10-12). In recent reports, stromal cell-derived factor- $1 \alpha$ (SDF- $1 \alpha)$ and its receptor CXCR4 have been recognized as providing generalized trophic support to both embryonic and mature neurons and helping support neurons damaged by injury or inflammation (13-15). Furthermore, SDF- $1 \alpha$ is a CXC chemokine produced by bone marrow stromal cells and is a potent chemoattractant for $\operatorname{HSCs}(16,17)$ that is constitutively expressed by most tissues (18).

In the nervous system, cellular prion protein $\left(\mathrm{PrP}^{\mathrm{C}}\right)$ is constitutively expressed in neurons and is abundant in regions such as the olfactory bulb, hippocampus, and synaptic neuropil in close spatiotemporal association with synapse formation. The physiological function of $\mathrm{PrPC}^{\mathrm{C}}$ remained obscure until several lines of prion gene-knockout mice were generated (19). Although these PrPnull mice were healthy throughout their life span, with normal development and resistance to scrapie infection (19), they were more susceptible to oxidative stress and apoptosis than wild-type mice (20). The localization of $\mathrm{PrPC}^{\mathrm{C}}$ in elongating axons suggests a role in axon growth $(21,22)$. Interestingly, interaction of $\operatorname{Pr}^{C}$ with the extracellular matrix protein laminin has been shown to promote neuritogenesis $(23,24)$.

In the present study, the cellular characteristics of hOECs/ONFs were studied in detail, including their immunophenotype, neuro- 

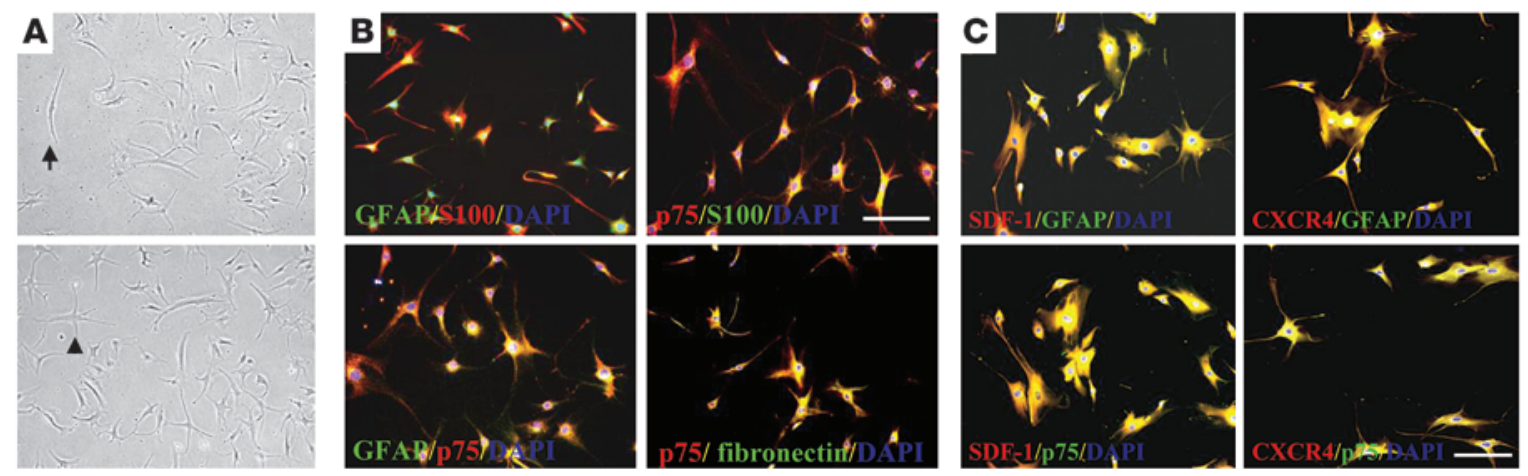

\section{Figure 1}

Phenotypic characterization of hOECs/ONFs. (A) The appearance of hOECs/ONFs by phase-contrast microscopy was either spindle shaped (arrow) or astrocyte-like (arrowhead). (B) Immunofluorescence colocalization analysis of hOECs/ONFs showed coexpression of p75 and GFAP, p75 and FN, p75 and S100, and GFAP and S100. (C) The proteins identified in our study as being produced by hOECs/ONFs including SDF-1 $\alpha$ and its receptor, CXCR4, were coexpressed with p75 and GFAP in a double immunofluorescence study in hOECs/ONFs. Scale bars: $50 \mu \mathrm{m}$.

trophic factor secretion, and signaling pathways under stress conditions. Using this cellular system, we intended to determine: (a) whether hOECs/ONFs could activate CXCR 4 and produce SDF- $1 \alpha$ in hypoxic and ischemic conditions; and (b) what role the interaction between soluble trophic factors such as SDF-1 $\alpha$ and cell receptors including $\mathrm{CXCR} 4$ and $\mathrm{PrP}^{\mathrm{C}}$ play in neuroregeneration in the ischemic brain. Specifically, transplantation of these olfactory accessory cells into stroke rats demonstrated that hOECs/ONFs enhance neuroplasticity, facilitate neurite outgrowth, and activate the stem cell homing process and thus promote reversal of the neurological deficit. In conclusion, this study demonstrates that hOECs/ONFs might potentially be of clinical benefit following autologous transplantation in stroke patients.

\section{Results}

Phenotypic characterization of hOECs/ONFs. hOECs/ONFs from surgical samples of nasal polyps were prepared and cultured on poly-D-lysine-coated chamber slides. They attached and grew slowly under standard culture conditions. The predominant cell morphology was spindle shaped, showing both a flattened fibroblast-like and an astrocyte-like pattern (Figure 1A). Immunocytochemical analysis consistently showed that at least 95\% of cells expressed both low-affinity nerve growth factor receptor (p75) and S100 antigen and a variable percentage of cells (30\%-70\%) expressed fibronectin (FN) and glial fibrillary acidic protein (GFAP). Double immunofluorescence analysis demonstrated that the hOECs/ONFs coexpressed p75/GFAP, p75/S100, p75/FN, and GFAP/S100 (Figure 1B): $94 \% \pm 2.8 \%$ of the cells expressed $S 100,95 \% \pm 3.3 \%$ of the cell population expressed $\mathrm{p} 75$, and $70 \% \pm 2.1 \%$ expressed GFAP.

hOECs/ONFs secrete SDF-1 $\alpha$ and upregulate CXCR4 under oxygen glucose deprivation treatment. In order to demonstrate the expression of SDF- $1 \alpha$ and its receptor CXCR4, double immunofluorescence examination, ELISA, and Western blot analysis with specific antibodies were performed in the hOECs/ONFs. The hOECs/ONFs coexpressed SDF- $1 \alpha$ and GFAP, SDF- $1 \alpha$ and p75, CXCR4 and GFAP, and CXCR4 and p75 (Figure 1C). The level of BDNF, GDNF, and VEGF in the hOEC/ONF medium under oxygen glucose deprivation (OGD) conditions, as determined by ELISA, was higher than that in control (data not shown). Levels of SDF- $1 \alpha$
(Figure 2A) and CXCR4 expression (Figure 2, B and C) also increased significantly 4 hours after OGD but fell to control levels over the next few hours. The corresponding cellular signaling pathways involved the activation of Akt and ERK1/2 one hour after OGD treatment (Figure 2, D and E), confirmed by the loss of increased SDF- $1 \alpha$ expression following the addition of specific inhibitors of activated Akt (LY294002) or activated ERK1/2 (PD98059) to treated cells (Figure 2F). The expression of p38 and JNK was not significantly altered by OGD (Figure 2, D and E).

hOECS/ONFs enhanced neurite regeneration and survival of primary cortical cultures after $O G D$. To evaluate whether soluble factors secreted from hOECs/ONFs enhanced the neurite regeneration and survival of primary cortical cultures (PCCs) after OGD, neurite process elongation and number of neurons surviving were measured in PCCs cocultured with hOECs/ONFs. Following OGD, significantly enhanced neurite length (Figure 3, A and B) and significantly more neurite-bearing neurons (Figure $3 \mathrm{~B}$ ) were found in hOEC/ONF-cocultured PCCs compared with control.

To confirm the correlation between neurite regeneration and $\mathrm{PrP}^{\mathrm{C}}$ expression, we performed Western blot and blocking antibody assays in a PCC and hOEC/ONF coculture system under OGD conditions. Western blot showed that expression of $\mathrm{PrPC}^{\mathrm{C}}$ in primary cortical neurons was significantly increased in PCCs cocultivated with hOECs/ONFs in comparison with PCCs alone (Figure 3C). Both the enhancement in neurite length and the increase in numbers of neurite-bearing neurons could be inhibited by addition of $\mathrm{PrP}^{\mathrm{C}}$-blocking antibody to the PCC coculture (Figure 3B).

$\operatorname{Pr} P^{C}$ interacts with CXCR4 in vitro. In order to characterize the possible association between $\mathrm{PrPC}^{\mathrm{C}}$ and CXCR4, PCCs cocultured with hOECs/ONFs were analyzed by double immunofluorescence immunohistochemistry (IHC) and IP with specific antibodies. IHC showed that CXCR4 was distributed in the cytoplasmic aggregates and concentrated in the perinuclear caps of the PCC (Figure 3D). The $\operatorname{PrP}^{\mathrm{C}}$ immunostaining appeared both in the cytoplasm and along neurites of the PCC (Figure 3D). Overlay of the IHC images for $\mathrm{PrP}^{\mathrm{C}}$ and $\mathrm{CXCR} 4$ showed that they coincided in the neurite and perinuclear regions of the PCC (Figure 3D).

In order to verify whether CXCR4 binds to $\mathrm{PrP}^{\mathrm{C}}$, we carried out co-IP analysis with specific antibodies for CXCR4 and PrPC. In the immunoprecipitated pellets, CXCR4 was seen to interact with $\mathrm{PrP}^{\mathrm{C}}$ 


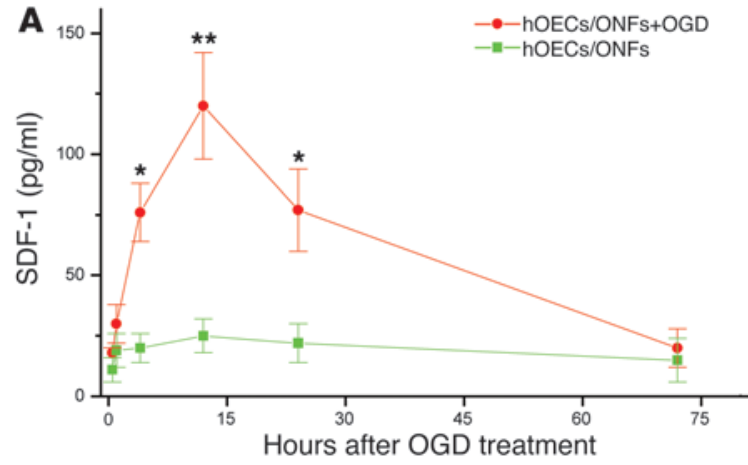

D
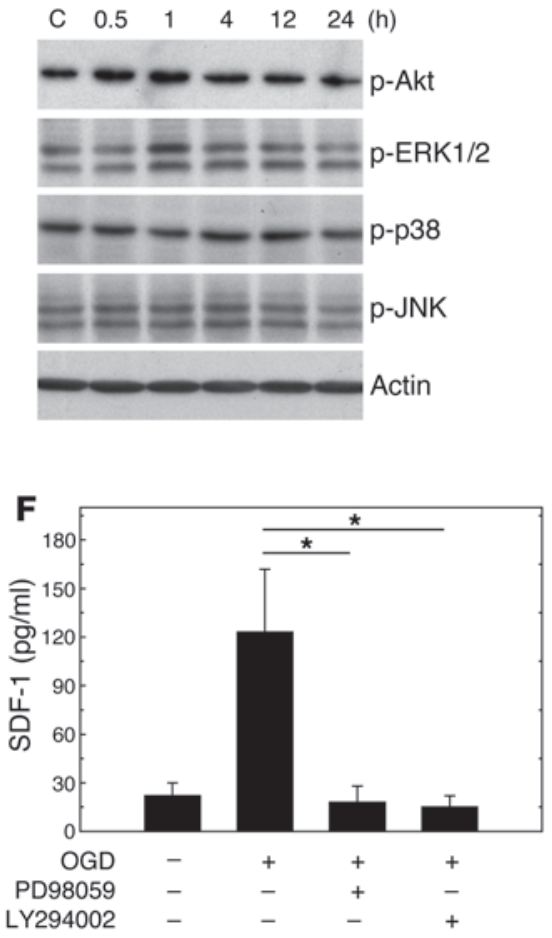

$\mathbf{E}$
$\mathbf{B}$

$\begin{array}{lllllll}\text { C } & 1 & 4 & 12 & 24 & 48 & (\mathrm{~h})\end{array}$
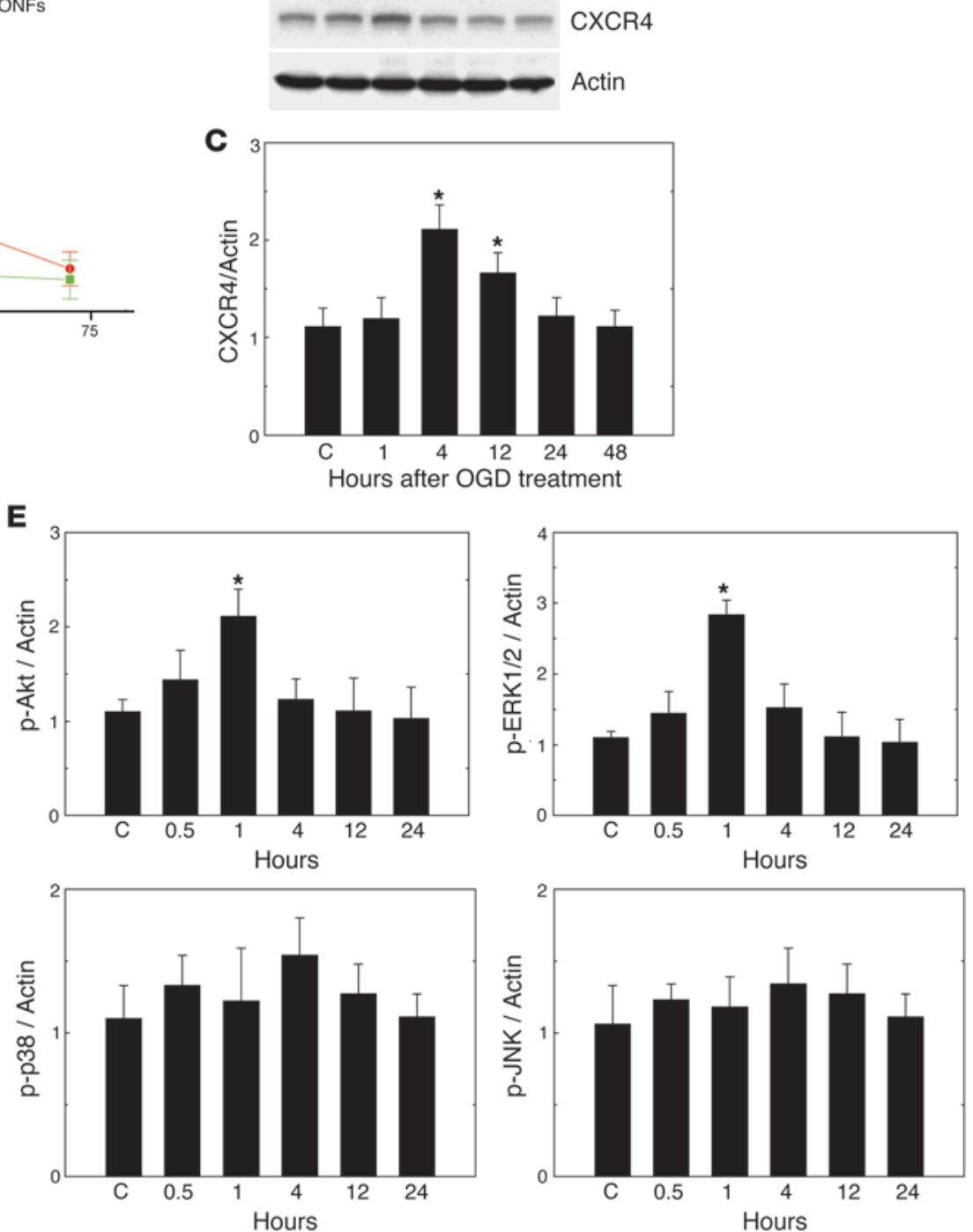

Figure 2

hOECs/ONFs upregulated the synthesis of soluble factors via the specific signaling pathway. (A-C) Under conditions of OGD, significantly increased expression of SDF-1 $\alpha$ and CXCR4 was found by ELISA and Western blot analyses, respectively. (D and E) Expression of signal transduction proteins (p-Akt and p-ERK1/2) increased significantly by 1 hour after treatment. (F) The upregulation of SDF-1 $\alpha$ could be blocked by specific inhibitors of $p$-Akt and p-ERK1/2 (LY294002 and PD98059). Data are expressed as mean \pm SEM. ${ }^{*} P<0.05$ and ${ }^{\star \star} P<0.01$ versus control.

(Figure 3E). These experiments indicate that $\operatorname{PrP}^{\mathrm{C}}$ does interact directly with CXCR4 in the PCC and hOEC/ONF coculture system. hOEC/ONF transplantation improves neurological dysfunction after cerebral ischemia. A body asymmetry trial and locomotor activity and grip strength assessments were used to assess whether implantation of hOECs/ONFs could improve neurological deficit after cerebral ischemia. Tests were conducted before and after middle cerebral artery (MCA) ligation in hOEC/ONF-treated rats and control rats. The behavioral measurement scores were all normalized to the baseline scores. Since unilateral cerebral ischemia causes imbalanced motor activity, all of the experimental rats developed significant body asymmetry, turning contralateral to the side of the induced ischemia on day 1 following cerebral ischemia. From
14 to 28 days after each treatment, rats with hOEC/ONF intracerebral transplants $(n=10)$ exhibited significantly reduced body asymmetry in comparison with control rats $(n=10)$ (Figure 4A). Locomotor activity was examined before and after cerebral ischemia in all animals. Vertical activity, vertical movement time, and the number of vertical movements significantly increased between 14 and 28 days after cerebral ischemia in rats receiving hOEC/ONF transplantation $(n=10)$ in comparison with control populations $(n=10)$ (Figure 4, B-D). Furthermore, measurement of forelimb grip strength for all experimental rats before and 28 days after treatment in each of the groups revealed a higher postischemia grip strength in the hOEC/ONF group $(n=10)$ than the control group $(n=10)$ (Figure 4E). 
A
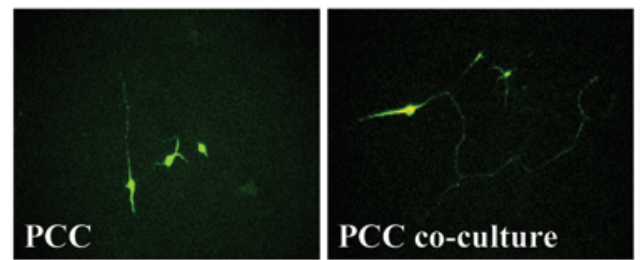

B
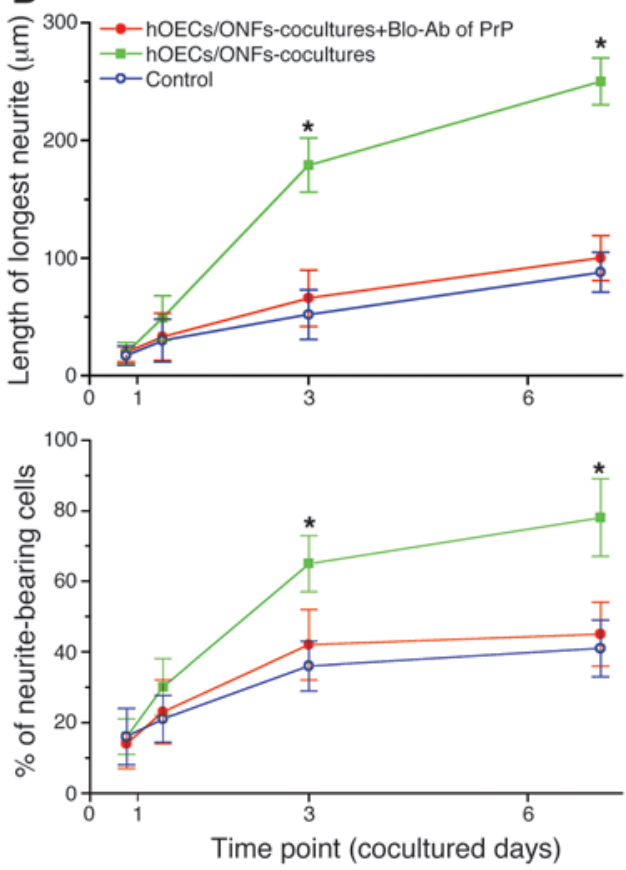

D
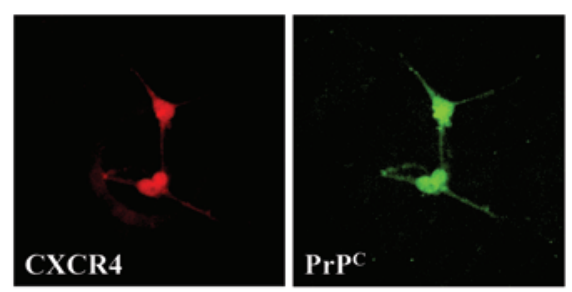

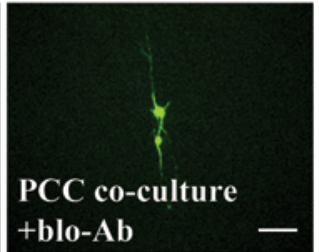

c
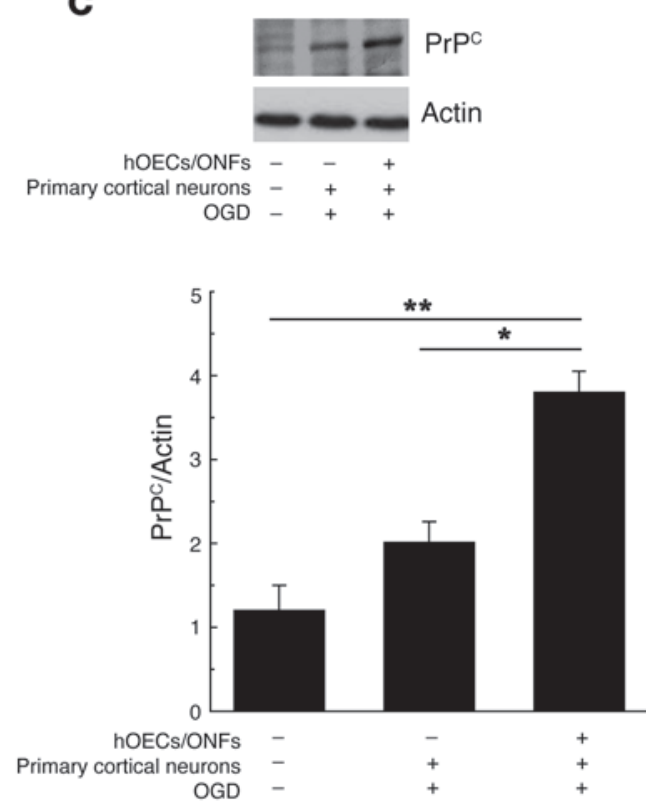

E

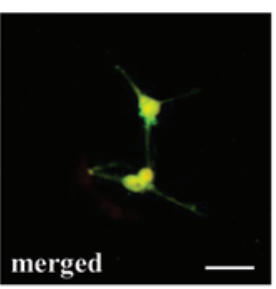

Figure 3

In coculture, hOECs/ONFs promoted neurite regeneration and survival of PCC under OGD. (A) Representative images of neurite regeneration in $\mathrm{PCC}$ with or without hOEC/ONF cocultivation. Blo-Ab, blocking Ab. (B) Neurite length and the number of surviving neurons increased in the hOECs/ONFs cocultured with PCC compared with the control. The enhancement of neurite length and number of surviving neurons was inhibited by the neutralization peptide of $\mathrm{PrPC}^{\mathrm{C}}$. (C) Upregulation of $\mathrm{PrPC}$ expression was found in PCC cocultured with hOECs/ONFs. (D) In IHC, subcellular colocalization of CXCR4 and $\mathrm{PrPC}^{\mathrm{C}}$ was shown by costaining with CXCR4 and $\mathrm{PrPC}$ antibodies in PCCs cocultured with hOEC/ ONFs. (E) Using coimmunoprecipitation analysis, the cell lysates were immunoprecipitated (IP) with anti-CXCR4 or anti-PrPC, and the signal of both proteins was detected by Western blotting (WB). Data are expressed as mean \pm SEM. ${ }^{\star} P<0.05$ and ${ }^{*} P<0.01$ versus control. Scale bars: $50 \mu \mathrm{m}$.
Enhancement of glucose metabolic activity in intracerebral hOEC/ONF transplantation group after cerebral ischemia. To verify whether hOEC/ ONF implantation could enhance glucose metabolic activity, experimental rats were examined by $\left[{ }^{18} \mathrm{~F}\right]$ fluoro-2-deoxyglucose PET (FDG-PET) using microPET. At 4 weeks after each treatment, the uptake of FDG was strikingly greater in the right cortical region (ischemic area of model) of the hOEC/ONF-treated group (Figure 4F). Semiquantitative measurement of relative glucose metabolic activity of the right cortical region revealed significant enhancement in the hOEC/ONF-treated $(n=6)$ compared with the control group $(n=6)$ (Figure 4F).

Intracerebral transplantation of hOECs/ONFs increased expression of growth factor and antiapoptotic proteins in vivo. In order to demonstrate whether the improvement in neurological function was correlated with soluble factors and survival agents after cerebral ischemia in hOEC/ONF-treated animals, we examined the expression of growth factor and antiapoptotic proteins in the ischemic cortical area using ELISA, immunohistochemical analysis, and Western blot analysis. Consistent with our immunohistochemical evidence, ELISA revealed a significantly increased level of SDF-1 $\alpha$ in the ischemic rats treated with hOECs/ONFs $(n=6)$ in comparison with vehicle-treated controls $(n=6)$ (Figure $4 \mathrm{G})$. In addition, significantly upregulated expression of antiapoptotic proteins Bcl-2 and Bcl-xL, but not Bax and Bad, was found in hOEC/ONFtreated rats $(n=6)$ at 3 days after cerebral ischemia compared with control rats $(n=6)$ (Figure 4, H and I).

Intracerebral hOEC/ONF transplantation stimulates endogenous stem cell mobilization, homing, and engraftment into rat brain following cerebral ischemia. BrdU labeling was used to follow the growth of these homing stem cells in the brain to determine whether intracerebral hOEC/ONF transplantation could enhance homing and engrafting of endogenous stem cells (from host brain and peripheral blood) into the damaged regions of the brain following ischemia. Cumulative BrdU labeling in hOEC/ONF transplantation rats revealed BrdU-immunoreactive cells in the ipsilateral hemisphere near the penumbra area, the striatal region (Figure 5, A-C), and the subventricular region of the lateral ventricle (Figure 5, D-F). BrdU-immunoreactive cells were also found around the lumen of 

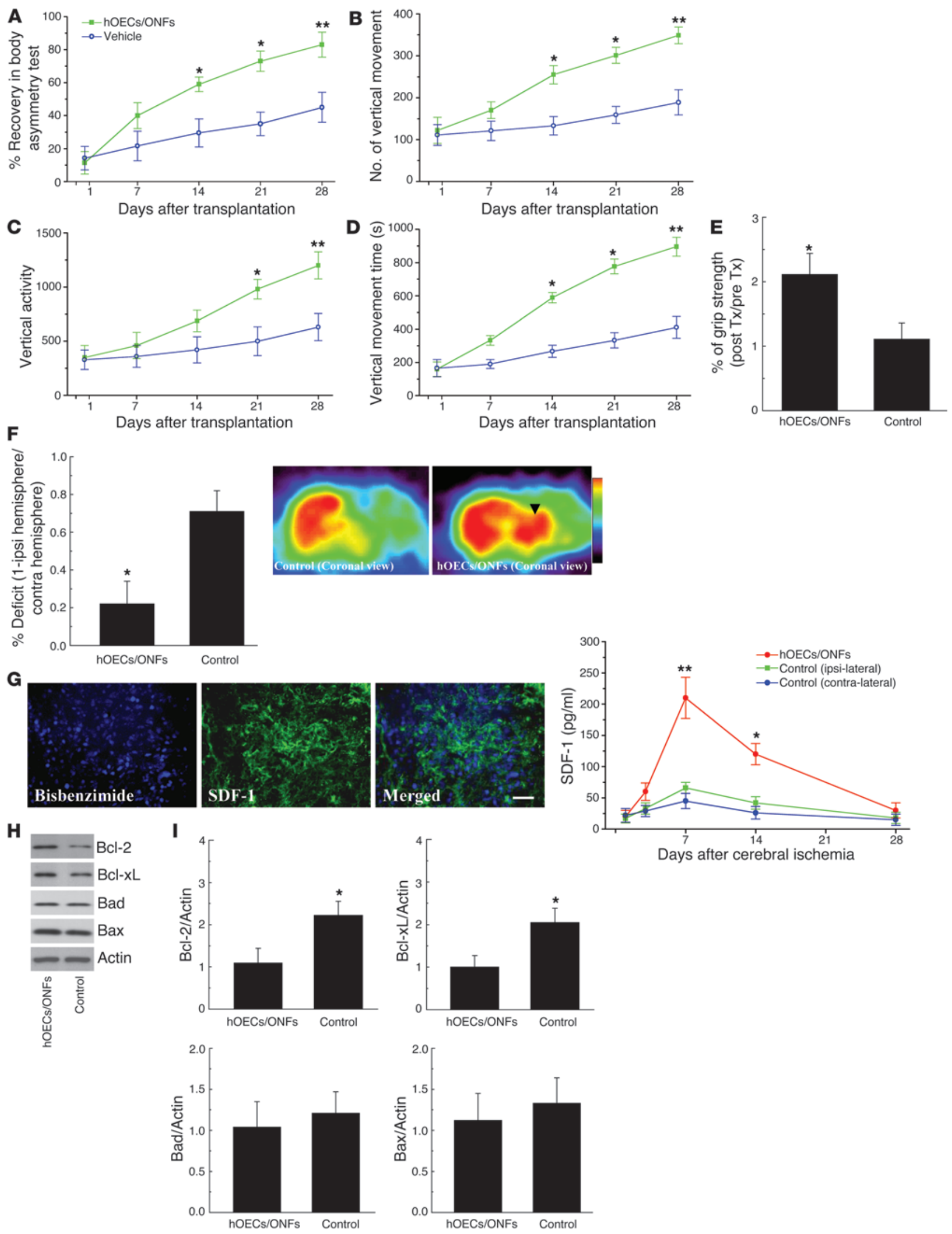


\section{Figure 4}

Intracerebral hOEC/ONF transplantation improves neurological behavior after cerebral ischemia. (A) A body asymmetry trial was used to assess body swing before and after MCA ligation. Between 14 and 28 days after cerebral ischemia, rats treated intracerebrally with hOECs/ ONFs exhibited significantly less body asymmetry than controls. (B-D) Locomotor activity of all experimental rats was examined. Vertical activity, vertical movement time, and the number of vertical movements significantly increased between 14 and 28 days after cerebral ischemia in rats receiving hOECs/ONFs in comparison with vehicle-treated control rats. (E) Grip strength measurement of the grasping power of forelimbs before (pre Tx) and 28 days after each of the 2 treatments (post $T x)$. There was a higher postischemia/preischemia strength ratio in the hOEC/ONF transplants than in vehicle control rats. (F) Representative result (coronal view) of FDG-PET of the right cortex (black arrowhead) of hOEC/ONF-treated and control groups. Semiquantitative measurement showed that relative glucose metabolic activity in the right cortex was much greater in the hOEC/ONF-treated than the control group (ipsi, ipsilateral; contra, contralateral). (G) In immunohistochemical and ELISA analyses, brain samples of the hOEC/ONF-treated rats showed significantly increased expression of SDF-1 $\alpha$ compared with the contralateral cortex and other areas of control rat brains. ( $\mathbf{H}$ and $\mathbf{I})$ In Western blot assay at 3 days after treatment, antiapoptotic proteins ( $\mathrm{Bcl}-2$ and $\mathrm{Bcl}-\mathrm{xL}$ ) were present at higher levels in the hOEC/ONFtreated than the control rat. Data are expressed as mean \pm SEM. ${ }^{*} P<0.05$ and ${ }^{* *} P<0.01$ versus control. Scale bar: $50 \mu \mathrm{m}$.

blood vessels of varying calibers (and also in endothelial cells of the vessel walls) (Figure 5, G-I) in the penumbral region. In BrdU pulse-labeling experiments, the number of BrdU-immunoreactive cells in the penumbral region of the ischemic cortex and striatum significantly increased in the hOEC/ONF-treated rats $(n=8)$ compared with saline-treated control rats $(n=8 ; P<0.05)$ (Figure $5 \mathrm{~J})$.

Intracerebral implantation of hOECs/ONFs could reconstruct the neural regeneration in the stroke model. To determine whether transplanted hOECs/ONFs and endogenous homing stem cells (from bone marrow stem cells [BMSCs] and INPCs) were responsible for the neuroplastic effect in the brains of hOEC/ONF-treated GFP-chimeric mice $(n=10)$ and BrdU-labeled mice $(n=10)$, the colocalization of cell type-specific markers at 28 days after transplantation was analyzed by double fluorescence IHC using laser scanning confocal microscopy (Figure 6A). There were significantly more BMSCs ( $\mathrm{GFP}^{+}$cells; green fluorescence) in the hOEC/ONF-treated GFP-chimeric mice than in controls $(n=10)$ (Figure 6A), and they were dispersed around the periphery of the transplanted hOECs/ONFs (Figure 6A). Moreover, there were also substantially more INPCs $\left(\mathrm{BrdU}^{+}\right.$nestin $\left.{ }^{+}\right)$in the hOEC/ONFtreated BrdU-labeled mice than in controls $(n=10)$ (Figure 6B). FISH analysis demonstrated that the implanted hOECs/ONFs labeled by bis-benzimide (blue fluorescence) were of human origin (Figure 6A). Interestingly, the implanted hOECs/ONFs and $\mathrm{GFP}^{+}$cells showed evidence of cell fusion (or nuclear fusion) (Figure 6C). About one-third of the hOECs/ONFs had fused with $\mathrm{GFP}^{+}$cells (binucleated cells) (Figure 6C). In a colocalization study (3D image) of exogenous transplanted hOECs/ONFs and endogenous homing stem cells, some bis-benzimide-labeled cells and some $\mathrm{GFP}^{+}$cells colocalized with microtubule associated protein $-2^{+}\left(\mathrm{MAP}^{+}{ }^{+}\right), \mathrm{vWF}{ }^{+}$, and $\mathrm{GFAP}^{+}$cells (red, neural cell-type specific markers) (Figure 6D) in the penumbra of hOEC/ONF-treated ischemic mouse brains. In addition, a few bis-benzimide-labeled hOECs/ONFs and $\mathrm{GFP}^{+}$cells costained for SDF-1 $\alpha$ immunoreactivity (Figure 6E).
Intracerebral bOEC/ONF implantation enhances neurite regeneration via coregulation of $\mathrm{PrP}^{\mathrm{C}}$ and $\mathrm{CXCR} 4$ expression. Neurite formation in the hOEC/ONF transplantation and control groups was measured to ascertain whether transplantation of hOECs/ONFs stimulated neurite outgrowth. Intracerebral hOEC/ONF transplantation substantially improved axonal regeneration in comparison with that in control rats (Figure 7A). Neurites extending over the penumbral areas and striatum were significantly longer in hOEC/ONF-treated $(n=8)$ than control rats $(n=8)$ at 28 days after cerebral ischemia (Figure $7 \mathrm{~B})$. Moreover, hOEC/ONF-treated rats $(n=8)$ had more neurite-bearing neurons in the penumbral areas and striatum at 28 days after cerebral ischemia than control rats $(n=8)$ (Figure 7B).

The possibility of a neuroplastic interaction between $\mathrm{PrPC}$ and CXCR4 induced by hOECs/ONFs was examined through immunofluorescence colocalization studies, Western blot analysis, and blocking antibody neutralization studies. In the double immunofluorescence study, CXCR4 and $\mathrm{PrP}^{\mathrm{C}}$ were coexpressed in the bis-benzimide-labeled hOECs/ONFs and $\mathrm{GFP}^{+}$cells in the GFP-chimeric mice after cerebral ischemia (Figure 7C). In addition, Western blot analysis showed a significant increase in expression of $\mathrm{PrP}^{\mathrm{C}}$ and CXCR4 in hOEC/ONF-treated $(n=6)$ compared with control rats $(n=6)$ (Figure $7 \mathrm{D})$. After addition of the $\mathrm{PrPC}^{\mathrm{C}}$ and CXCR4 blocking/neutralizing antibodies, the degree of neurite regeneration $(n=12)$ (Figure 7B) and the neurological behavior measurements $(n=12)$ (Figure $7 \mathrm{E})$ indicated no significant differences among the 3 therapeutic groups (hOECs/ONFs with $\mathrm{PrP}^{\mathrm{C}}$-blocking antibody; hOECs/ONFs with CXCR4 neutralizing antibody; and hOECs/ONFs with control human IgG). However, hOEC/ONF implantation did not significantly reverse the neurite degeneration in the $\operatorname{Pr} P^{C}$-knockout $\left(\operatorname{Pr} P^{o / o}\right)$ mice $(n=8)$ compared with that of $\mathrm{PrP}^{+/+}$mice $(n=8)$ after cerebral ischemia (Figure 7F).

\section{Discussion}

Although many studies have focused on OECs with regard to reversal of demyelination and axonal degeneration such as in spinal cord injury $(3,25-27)$, few reports have looked at the ability of OECs to repair ischemic neural injuries. In previous studies, it has been demonstrated that the olfactory epithelium (OE), which is highly vulnerable to injury, is endowed with a constitutive capacity for progenitor cell proliferation to reconstruct damaged olfactory neurons $(28,29)$. Furthermore, recent reports have shown that $\mathrm{OE}$ can induce simple neurogenesis following direct damage caused by exposure to methyl bromide gas $(30,31)$. This neurogenesis might be facilitated by factors including Mash1 (32) and Ngn1 (33) and enhance the proliferation of progenitor cells in the olfactory system. Therefore, in this report, we intended to re-verify the neuroplastic capacity of hOECs/ONFs using a different stress model of hypoxia/ischemia in both PCC and a rat stroke model. First, in view of the role of trophic factors in neuroprotection, the constitutive synthesis of many growth factors by the olfactory system indicated that it would be useful to elucidate how these factors contribute to survival of the injured neurons and regulate nervous system development (34). The most important neurotrophic factors secreted from the olfactory pathway are BDNF (35), GDNF (36), HGF (37), and SCF (38). In our study, we also found that the level of BDNF, GDNF, and VEGF significantly increased in hOEC/ONF medium after OGD and we showed that SDF- $1 \alpha$ was found both in the cultured hOEC/ ONF medium after OGD and in the hOEC/ONF-transplanted ischemic brain. The corresponding SDF- $1 \alpha$ receptor, CXCR4, 

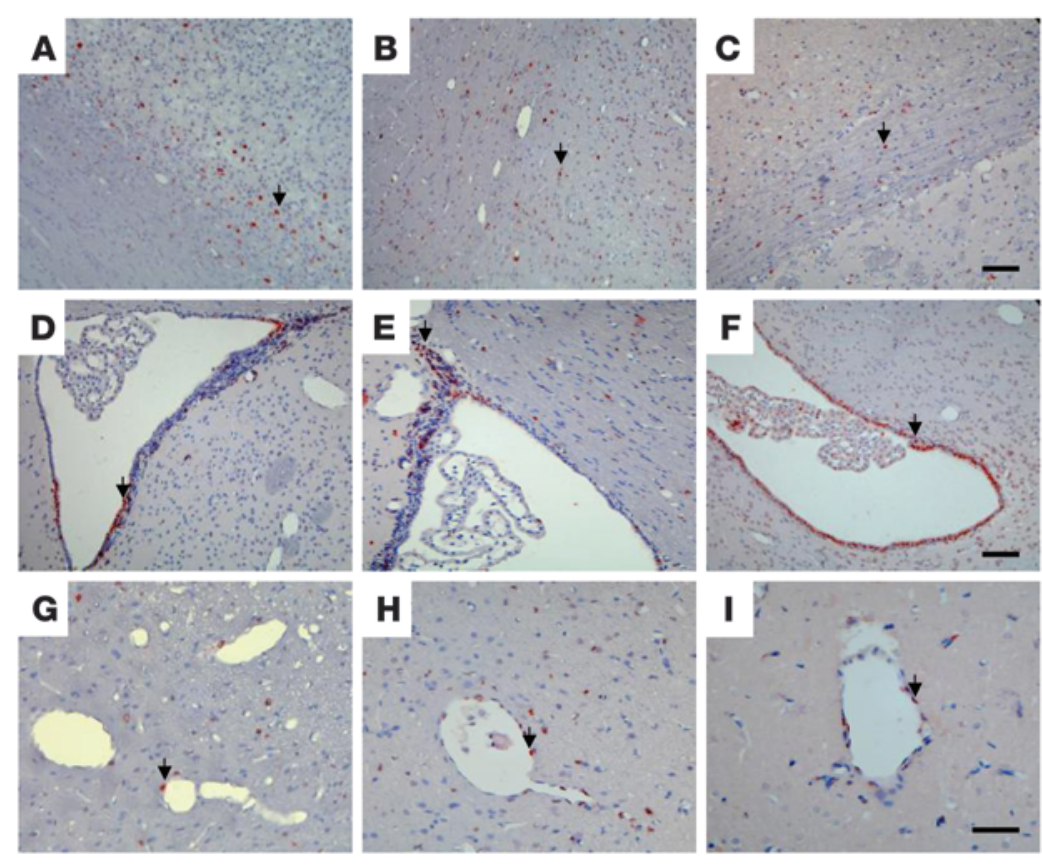

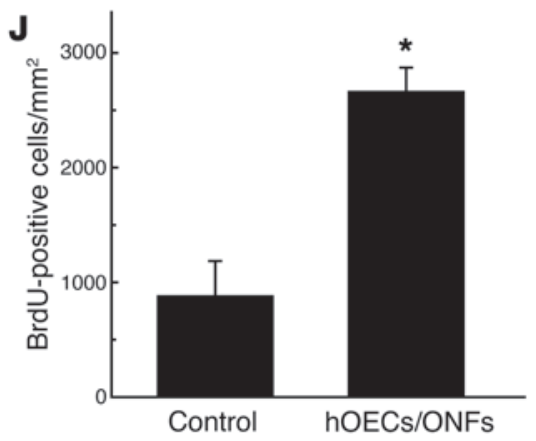

Figure 5

Immunohistochemical staining for BrdU in rats treated with hOECs/ONFs after cerebral ischemia. A few BrdU-immunoreactive cells (arrows) were detected around the infarct boundary $(\mathbf{A}-\mathbf{C})$, in the subventricular area (D-F), and over the perivascular region $(\mathbf{G}-\mathbf{I})$ in the ipsilateral cortex. (J) Quantitative analysis revealed that the number of BrdU-immunoreactive cells in the ipsilateral hemisphere of rats treated with hOECs/ONFs was significantly increased at 28 days after treatment in comparison with rats treated with vehicle control. Data are expressed as mean \pm SEM. ${ }^{*} P<0.05$ versus control. Scale bars: $50 \mu \mathrm{m}$.

was also highly expressed in the hOECs/ONFs. Since SDF- $1 \alpha$ could be secreted from specific glial cells under certain conditions $(13,39)$, one can assume that hOECs/ONFs share features in common with glial cells (40) and can secrete SDF-1 $\alpha$ under conditions of hypoxia/reoxygenation. Furthermore, there is also some evidence suggesting increased expression of SDF- $1 \alpha$ and CXCR 4 in cases of neuropathogenesis induced by many forms of injury, including trauma, stroke, and inflammation $(14,15,41)$. Therefore, SDF- $1 \alpha$ might not only provide generalized trophic support to both embryonic and mature neurons, but also help support neurons damaged by injury or inflammation. In addition, in a recent report, SDF- $1 \alpha$ was found to play a critical role as a guidance molecule regulating neurite outgrowth in cultured cerebellar granular neurons (42). However, even if expression of SDF- $1 \alpha$ was downregulated in the hOEC/ONF implantation, hOECs/ONFs could still produce other factors to rescue the injured neurons. In this study, hOECs/ONFs transduced the neuroprotective signal through direct upregulation of antiapoptotic proteins (Bcl-2 and $\mathrm{Bcl}-\mathrm{xL}$ ) and enhancement of neurite regeneration, as well as via the known soluble trophic factors BDNF, GDNF, and VEGF. Therefore, SDF-1 $\alpha$, one of the major trophic factors secreted by hOECs/ONFs, might also play a significant role in neural survival and regeneration in our hypoxia/ischemia model.

In a recent report, Beites et al. concluded that neural stem cells resident in the OE could be regulated dynamically to induce neurogenesis during development and enhance regeneration after injury (43). In fact, one of the major components of OE stem and progenitor cells was OECs (43). Many reports have demonstrated that OECs can rebuild a demyelinated axon via production of a
Schwann cell-like sheath in the spinal cord of an injured animal $(3,6,44)$. Subsequent to the remyelination, OECs also replaced the myelin to regenerate disconnected axons and finally restored the neural conductance of the corticospinal tract $(45,46)$. Furthermore, OEC transplantation also stimulated neurite regrowth to recover body movement in spinal cord injury $(47,48)$.

In this report, we present the first evidence to our knowledge that implantation of hOECs/ONFs enhances homing of endogenous stem cells, from peripheral blood and host brain, into the ischemic brain. Recent reports indicate that SDF-1 $\alpha /$ CXCR4 is a strong chemoattractant for stem cells and also plays a significant role in endogenous stem cell trafficking between peripheral circulation and bone marrow (49). A further study indicated that focal cerebral ischemia causes an upregulation in SDF-1 $\alpha$ / CXCR4 expression over the peri-infarct area (13). This induction of upregulation of endothelial SDF-1 $\alpha(50)$ and the appearance of increased CXCR4 expression in the ischemic hemisphere after ischemia explained in our previous report (51) together indicate that SDF-1 $\alpha$ secreted from the implanted hOECs/ONFs in this study could be a chemoattractant for endogenous stem cells. Therefore, exogenous implanted hOECs/ONFs and endogenous homing stem cells might coordinate to exert a neuroplastic effect to repair the injured brain.

In recent reports, OECs have been proven to promote neurite regrowth in vitro (48), and secreted neurotrophic factors and cell-cell contact mechanisms are probably involved in the neurite regeneration (48). Although previous reports have demonstrated that growth factors secreted from OECs such as nerve growth factor (NGF), BDNF, GDNF, and neurturin (NTN) might indirectly 
A
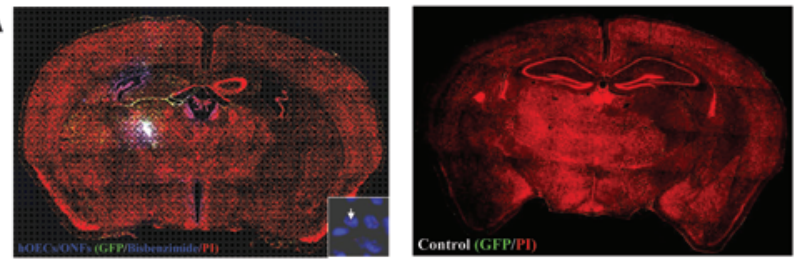

B

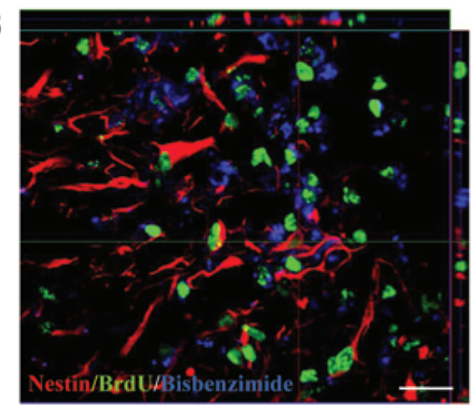

D
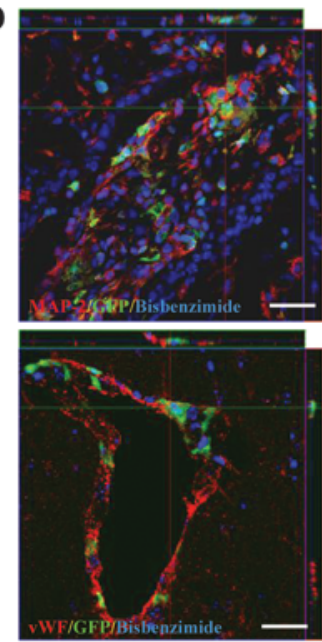

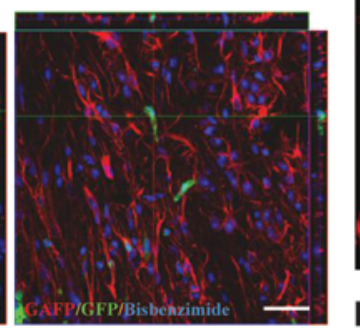

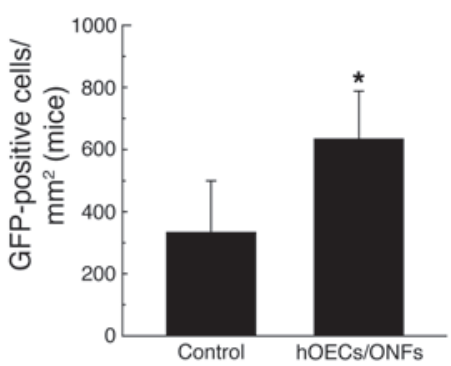

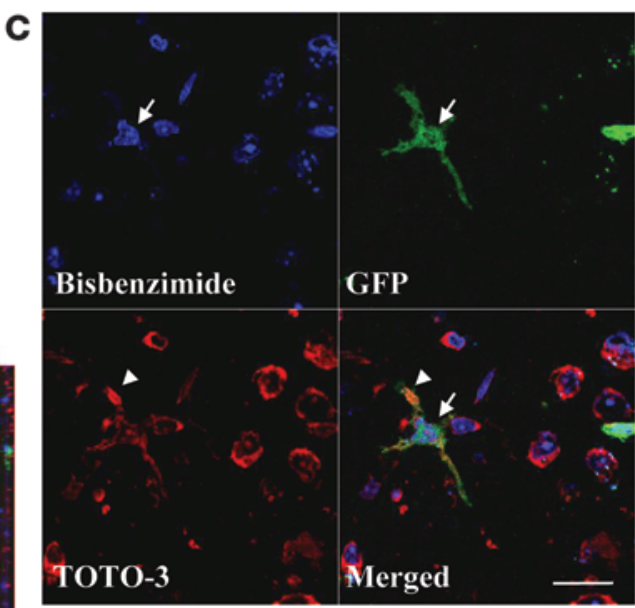

E

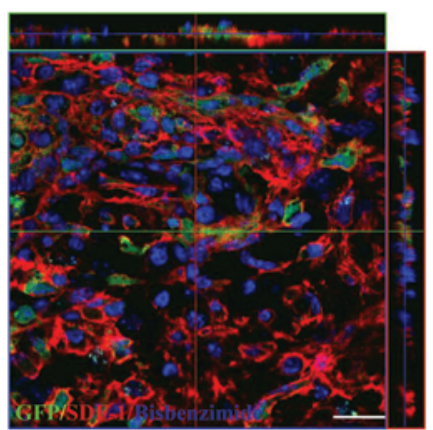

Figure 6

Biological mechanism of neuroplastic effects on the ischemic brain after intracerebral transplantation of hOECs/ONFs. (A) In a representative brain section of a GFP-chimeric mouse treated with or without hOECs/ONFs (white arrow indicates the injection site), GFP+ cells are seen dispersed over the periphery of the transplanted hOECs/ONFs and were significantly increased in quantity in the hOEC/ONF-treated mice in comparison with controls. In FISH analysis (white arrow, 2 red spots), hOECs/ONFs were shown to be of human origin (inset square in left panel). (B) IHC of hOEC/ONF treatment in the BrdU-labeled mice. Many BrdU+nestin ${ }^{+}$cells were distributed around the transplanted hOECs/ONFs. (C) Interestingly, 1 cell with 2 nuclei (cell fusion) was found in the implanted hOECs/ ONFs (white arrows, blue nucleus) and $\mathrm{GFP}^{+}$cells (white arrowheads, red nucleus). The nucleic dye TOTO-3 (red) was used to define the outline of all nuclei in the section. (D) In a colocalization study (3D image) some bis-benzimidelabeled cells and some GFP+ cells colocalized with MAP-2+, vWF+, and $\mathrm{GFAP}^{+}$cells in the penumbra of hOEC/ONF-treated ischemic rat brains. (E) SDF-1 $\alpha$-immunoreactive cells colocalized with a few bisbenzimide-labeled hOECs/ONFs and $\mathrm{GFP}^{+}$cells. Data are expressed as mean \pm SEM. ${ }^{*} P<0.05$ versus control. Scale bars: $50 \mu \mathrm{m}$. facilitate axon regeneration (52), the actual molecules involved in the cell-cell contact mechanism that directly mediated the neurite outgrowth-stimulating effect of hOECs/ONFs are not completely known. Some investigations have found that cell adhesion molecules, such as L1 and N-cadherin, were associated with the neurite outgrowth $(53,54)$. Furthermore, it is known that $\operatorname{Pr}^{\mathrm{C}}$ plays an important role in the regulation of neurite regeneration (23). In a recent study, some proteins in the living brain involved in cell adhesion and neurite outgrowth were found in the presence of $\operatorname{PrP}^{\mathrm{C}}(55)$. Therefore, $\operatorname{PrP}^{\mathrm{C}}$ must interact with some intercellular matrix proteins to facilitate neurite regeneration. For example, $\mathrm{PrP}^{\mathrm{C}}$ participated in neurite adhesion through its interaction with laminin (24). In addition, the laminin receptor, which is a critical factor for cell differentiation and proliferation, was also identified as a cell-surface binding companion of $\operatorname{PrP}^{\mathrm{C}}(56)$. Since laminin contributes to axon growth and fiber tract formation, $\operatorname{PrP}^{\mathrm{C}}$ is particularly relevant for neurite formation during brain development (57). Although the SDF-1 $\alpha /$ CXCR 4 signaling pathway has also been shown to regulate axonal elongation (58) and guide the neu- rite growth cone $(59,60)$, few reports have investigated the interaction between CXCR4 and $\operatorname{PrPC}^{\mathrm{C}}$ in neural regeneration (61). The combination of SDF-1 $\alpha /$ CXCR 4 with laminin might modulate cellular migration (62). In this study, we have demonstrated for the first time to our knowledge that coexpression of CXCR4 and $\mathrm{PrP}^{\mathrm{C}}$ might enhance the neurite growth in the cellular component of hOECs/ONFs cocultured with PCC under OGD conditions and in the brain of hOEC/ONF-implanted stroke animals. It is possible that these 2 proteins interact in trans with each other, that is, they facilitate an interaction between the cell surface of one cell and the extracellular protein matrix or the surface of an adjacent cell. Furthermore, investigation using blocking antibodies against CXCR4 and $\mathrm{PrP}^{\mathrm{C}}$ showed inhibition of neurite regeneration and even neurological function recovery.

In general, one of the major mechanisms by which bone marrow stem/progenitor cells repair tissue injury is cell/nuclear fusion (63-65). Terada et al. and Ying et al. investigated spontaneous fusion between embryonic stem cells (63) and neural stem cells (64). The frequency of cell/nuclear fusion ranged from $10^{-4}$ per 

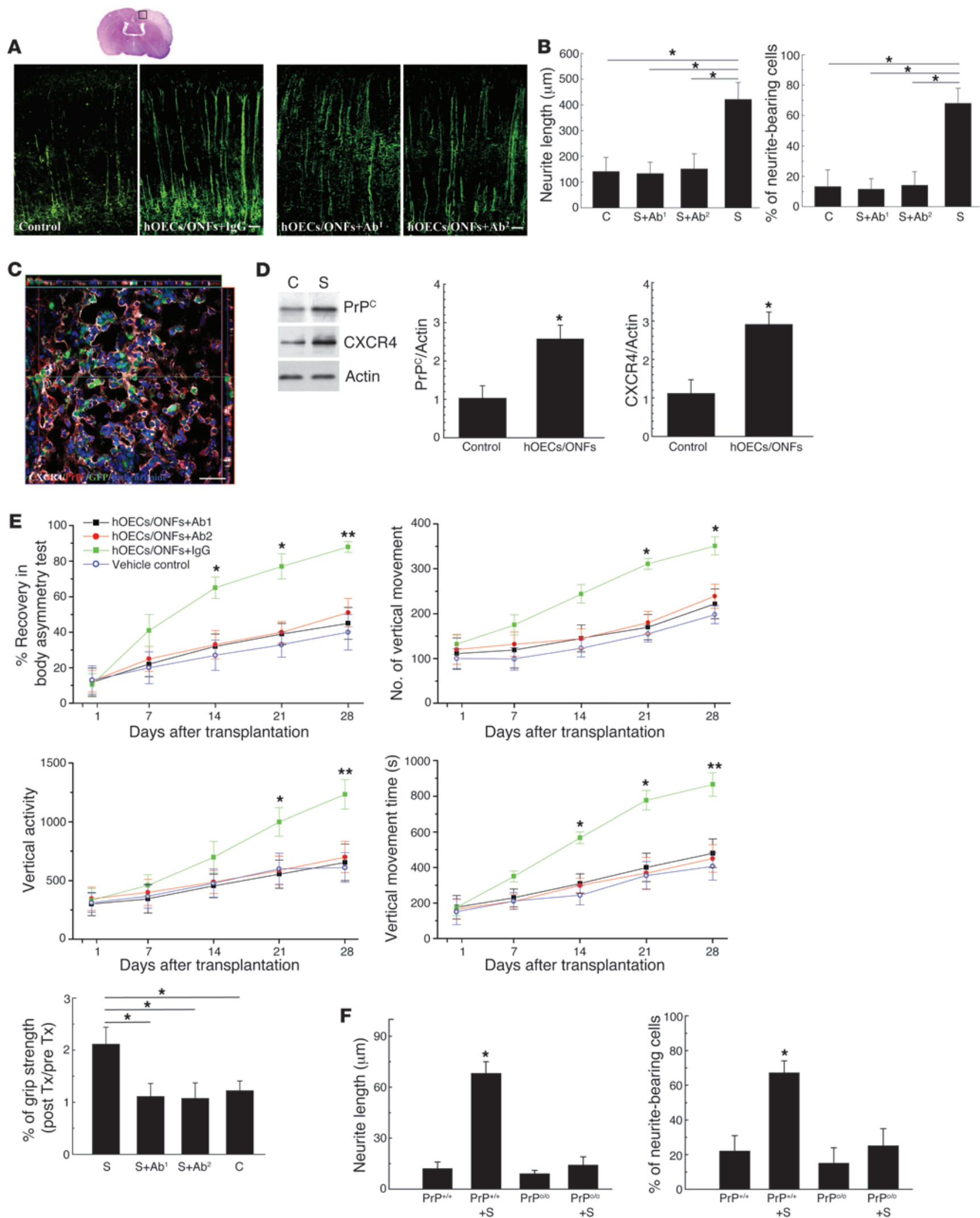


\section{Figure 7}

Intracerebral transplantation of hOECs/ONFs enhanced neurite regeneration through upregulation of $\mathrm{PrPC}$ and $\mathrm{CXCR} 4$ expression. (A) In immunohistochemical analysis, hOEC/ONF + IgG (control human IgG) implantation significantly improved neurite outgrowth in the penumbral area of rats in comparison with hOECs/ONFs plus $\mathrm{Ab}^{1}$ (PrPC-blocking antibody), hOECs/ONFs plus $\mathrm{Ab}^{2}$ (CXCR4-neutralizing antibody), and vehicle (Control) treatment. (B) hOEC/ONF + IgG (S) implantation produced neurites significantly longer than those found in rats treated with $S+A b^{1}, S+A b^{2}$, and vehicle (control [C]). Moreover, hOEC/ONF + IgG-treated rats had more neurite-bearing neurons than the control rats. (C) In colocalization analyses, $\mathrm{PrPC}$ and CXCR4 coexpressed around the region of bis-benzimide-labeled hOECs/ONFs and GFP+ cells on the cerebral ischemic microenviroment. (D) In a Western blot analysis, $\mathrm{PrPC}$ and $\mathrm{CXCR} 4$ expression was upregulated in the hOEC/ ONF-treated rats compared with control rats. (E) The neurological behavior measurement modalities showed no significant difference between the 3 therapeutic groups $\left(C, S+A b^{1}, S+A b^{2}\right)$. (F) In evaluating neurite regeneration, hOEC/ONF implantation in the $\mathrm{PrPo} / \mathrm{o}$ mice did not increase the neurite length and number of neurite-bearing cells in contrast to those of $\mathrm{PrP}^{+/+}$mice after cerebral ischemia. Data are expressed as mean \pm SEM. ${ }^{\star} P<0.05$ and ${ }^{\star \star} P<0.01$ versus control. Scale bars: $50 \mu \mathrm{m}$.

cell plated $(63,64)$ to one-quarter of cells $(66)$. They concluded that the phenomenon of cell/nuclear fusion was a frequent rather than a rare event. In our study, we demonstrated that cell/nuclear fusion occurred between hOECs/ONFs and BMSCs. Although a higher percentage of fused cells was found in this model than in previous investigations (66), the other 2 mechanisms also correlated well with stem cell-induced neuroplasticity in this study, including differentiation into tissue-specific phenotypes and secretion of many trophic factors.

\section{Methods}

Separation and culture of hOECs/ONFs. Human nasal polyp (hNP) samples $\left(5 \mathrm{~mm}^{3}, 0.5 \mathrm{~g}\right.$ in weight) were collected in sterile boxes containing HBSS (Gibco/BRL; Invitrogen), for primary culture within 24 hours. Protocols for sampling hNP were approved by the Institutional Review Board of China Medical University and Hospital. Written informed consent was obtained from all patients. In brief, the donated tissue was dissected into small pieces under a dissecting microscope and placed in a phosphate-buffered solution at room temperature. The tissue was then ground with a dissection scalpel and transferred into $10 \mathrm{ml}$ DMEM/F12 medium containing trypsin and EDTA and shaken at $37^{\circ} \mathrm{C}$ in a water bath for 5 minutes. It was then rinsed with DMEM/F12 solution and triturated with a fire-polished Pasteur pipette. The ground tissue explants were collected by centrifugation at $600 \mathrm{~g}$ for 10 minutes. The resulting pellet was resuspended in DMEM/ F12 medium containing B27 medium supplement, 10\% FCS, and 1\% $(100 \mathrm{U} / \mathrm{ml})$ penicillin/streptomycin at $3 \times 10^{5}$ cells per ml culture medium. The tissue was placed in a $75-\mathrm{cm}^{2}$ flat flask and incubated in $5 \% \mathrm{CO}_{2}$ at $37^{\circ} \mathrm{C}$. The tissue was left undisturbed for 5-7 days to allow for migration of the cells from the explants. These primary cells were passaged again once a week for 3-4 weeks.

Immunocytochemical analysis and quantitative assessment of antigenicity in hOECs/ONFs. Immunocytochemical studies of the hOECs/ONFs were performed using different antibodies: p75 (1:100; Millipore), GFAP (1:300; Millipore), FN (1:1,000; Molecular Probes; Invitrogen), S100 (1:1,000; Dako), oligodendrocyte marker 4 (O4; 1:100; Millipore), SDF-1 $\alpha$ (1:200; Torrey Pines Biolabs), CXCR4 (1:200; Torrey Pines Biolabs), neurofilament-200 (NF-200; 1:300; Sigma-Aldrich), $\beta$ III-tubulin (Tuj-1, 1:200; Mil- lipore), MAP-2 (1:300; Millipore), and neuronal nuclear antigen (Neu-N; 1:50; Millipore). Cells were plated on a poly-L-lysine-coated chambered glass slide at a standard density and allowed to grow at $37^{\circ} \mathrm{C}$ in $5 \% \mathrm{CO}_{2}$ for 24 hours. The antigenicity of the cell population was quantitated as previously described with modification (67). In brief, a random field of view was selected at $\times 200$ magnification with the UV filter. Images were captured and assessed using a spot digital camera (Diagnostic Instruments) and Northern Eclipse 5.0 software (Empix).

Total protein extraction, Western blot assay, and ELISA in vitro. For OGD treatment, the hOECs/ONFs were cultured with glucose-free Earle's balanced salt solution, placed in a hypoxic chamber (Bugbox; Ruskinn) for 4 hours, and continuously flushed with $95 \% \mathrm{~N}_{2}$ and $5 \% \mathrm{CO}_{2}$ at $37^{\circ} \mathrm{C}$ to maintain a gas-phase $\mathrm{PO}_{2}$ of less than $1 \mathrm{mmHg}(\mathrm{OM}-14$ oxygen monitor; SensorMedics). Control cells were incubated in glucose-free Earle's balanced salt solution in a normoxic incubator for the same period. OGD was terminated by switching back to normal culture conditions. After OGD treatment, the cells were returned to a $37^{\circ} \mathrm{C}$ normoxic incubator $\left(95 \%\right.$ air and $\left.5 \% \mathrm{CO}_{2}\right)$ for different time periods ( 30 minutes, 1 hour, 4 hours, 12 hours, 24 hours, and 48 hours) of reoxygenation. The hOECs/ONFs were then collected at each time point and stored at $-80^{\circ} \mathrm{C}$ for protein extraction. Western blot analyses of hOECs/ONFs were performed after hypoxia-reoxygenation treatment. Briefly, hOECs/ONFs were lysed in a buffer containing $320 \mathrm{mM}$ sucrose, $5 \mathrm{mM}$ HEPES, $1 \mu \mathrm{g} / \mathrm{ml}$ leupeptin, and $1 \mu \mathrm{g} / \mathrm{ml}$ aprotinin. Lysates were centrifuged at $13,000 \mathrm{~g}$ for 15 minutes. The resulting pellet was resuspended in sample buffer ( $62.5 \mathrm{mM}$ Tris- $\mathrm{HCl}, 10 \%$ glycerol, $2 \%$ SDS, $0.1 \%$ bromophenol blue, and $50 \mathrm{mM}$ DTT) and subjected to SDS-PAGE (4\%-12\%). Proteins in the gel were then transferred to a Hybond-P nylon membrane. This was followed by incubation with appropriately diluted antibodies against CXCR4 (1:100; Millipore), $\operatorname{PrPC}$ (1:300; M20; Santa Cruz Biotechnology Inc.), activated Akt (1:200; Calbiochem), activated ERK1/2 (1:200; Santa Cruz Biotechnology Inc.), activated p38 (1:200; Santa Cruz Biotechnology Inc.), activated JNK (1:200; Santa Cruz Biotechnology Inc.), and $\beta$-actin (1:2,000; Santa Cruz Biotechnology Inc.). Specific ERK1/2 pathway inhibitor PD98059 (10 $\mu \mathrm{M}$; Cell Signaling Technology) and Akt pathway inhibitors wortmannin or LY294002 (10 nM; Calbiochem), which were used to pretreat cells, were applied to the hOECs/ONFs to suppress enzyme binding in order to block the transcriptional signal of ERK1/2 and Akt. Membrane blocking, primary and secondary antibody incubations, and chemiluminescence reactions were conducted for each antibody individually according to the manufacturer's protocol. The intensity of each band was measured using a Kodak Digital Science 1D Image Analysis System. In addition, the total amount of growth factors in the medium was measured with a Quantikine ELISA kit (R\&D Systems) according to the manufacturer's instructions. Optical density was measured using a spectrophotometer (Molecular Devices), and standard curves were generated with the program SoftMax (Molecular Devices).

In vitro PCC preparation and coculture with hOECs/ONFs. PCC cells were prepared from the cerebral cortex of gestation day-17 embryos from SpragueDawley rats as described previously (68). In brief, pooled cortical tissues isolated from the brain were dissociated by mechanical trituration in HBSS. Then, cells were counted and plated at a density of $5 \times 10^{5}$ cells in 24-well culture plates precoated with poly-D-lysine $(0.02 \mathrm{~g} / \mathrm{l})$. Cells were grown in DMEM (Gibco-BRL; Invitrogen) with $10 \%$ heat-inactivated FBS, $1 \mathrm{mM}$ pyruvate, $4.2 \mathrm{mM}$ sodium bicarbonate, $20 \mathrm{mM}$ HEPES, and antibiotics. Cultures were maintained at $37^{\circ} \mathrm{C}$ in a humidified atmosphere of $5 \% \mathrm{CO}_{2}$ and $95 \%$ air. Four days after isolation, the cultures were replenished with MEM (Gibco-BRL; Invitrogen) containing $0.5 \mathrm{~g} / 1 \mathrm{BSA}$ and $\mathrm{N}-2$ supplement, $0.5 \mathrm{mM}$ pyruvate, and antibiotics. Finally, the culture medium was changed to a serum-free MEM containing $1 \mathrm{mM}$ pyruvate, $1 \mathrm{mM}$ glutamate, $0.5 \mathrm{~g} / 1$ BSA, $0.3 \mathrm{mM} \mathrm{KCl}$, and antibiotics on the seventh day of incubation. 
During the coculture procedure, the culture medium was removed from hOEC/ONF culture and replaced with a $1.5-\mathrm{ml}$ cell suspension containing $2 \times 10^{5} \mathrm{PCC}$ cells. These cocultures were maintained for 3 days in the same culture condition. For OGD treatment, the cocultured cells were incubated with glucose-free Earle's balanced salt solution, placed in a hypoxic chamber (Bugbox) for 4 hours, and continuously flushed with $95 \% \mathrm{~N}_{2}$ and $5 \% \mathrm{CO}_{2}$ at $37^{\circ} \mathrm{C}$ to maintain a gas-phase $\mathrm{PO}_{2}$ of less than $1 \mathrm{mmHg}$ (OM-14 oxygen monitor; SensorMedics). After OGD treatment, the cocultured cells were returned to a $37^{\circ} \mathrm{C}$ normoxic incubator $(95 \%$ air and $5 \%$ $\mathrm{CO}_{2}$ ) for different time periods (1, 3, and 7 days) of reoxygenation, before immunostaining and Western blot analysis.

Immunocytochemistry and assessment of neurite regeneration. For $\beta$-tubulin immunostaining, cell cultures were washed with PBS and fixed for 30 minutes at room temperature in $4 \%$ paraformaldehyde. After washing in PBS, the fixed cultured cells were treated for 30 minutes with blocking solution (10 g/l BSA, $0.03 \%$ Triton X-100, and $4 \%$ serum in PBS). Cells were incubated overnight at $4{ }^{\circ} \mathrm{C}$ with an antibody against $\beta$-tubulin (1:200; Millipore) for 3 hours, followed by a secondary antibody conjugated with FITC for 1 hour, and then rinsed 3 times in PBS. Finally, the slides were lightly counterstained with DAPI, washed with water, and then mounted.

The number of neurite-bearing cells and neurite length were assessed as previously described previously, with modification (69). In brief, cells in each treated group were plated after OGD, fixed, and immunostained for $\beta$-tubulin. For quantification, neurons with neurites were defined as those bearing a process greater than twice the cell body length. The length of the longest neurite of each neuron was measured from digitized images and quantified using the SigmaScan imaging analysis program (SigmaScan 4.01.003). All measurement data were calculated from experiments performed in triplicate.

To assess the correlation between neurite regeneration and $\mathrm{PrPC}^{\mathrm{C}}$ expression, we evaluated the neurite length and number of neurite-bearing cells in the presence of prion protein-blocking antibody (6H4; Prionics) under OGD conditions. The primary cortical neurons were pretreated with $\mathrm{PrP}_{-}$ blocking antibody $(6 \mu \mathrm{g} / \mathrm{ml})$ and then cocultured with OECs for about 40 hours (70), before the cocultured cells received OGD treatment and neurite regeneration was assessed as outlined above.

Coimmunoprecipitation analysis. The immunoprecipitation experimental procedures were as previously described (71). First, cells were managed with lysis buffer $(50 \mathrm{mM}$ Tris-HCl, pH 7.5, 1\% NP-40, $150 \mathrm{mM} \mathrm{NaCl}$, $0.5 \%$ sodium deoxycholate, and protease inhibitor). The lysate $(300 \mu \mathrm{g})$ was incubated with protein $\mathrm{A} / \mathrm{G}$-agarose beads at $4{ }^{\circ} \mathrm{C}$ for 6 hours. Then, CXCR4 (mAb 173; R\&D Systems) and PrPC (C-20; Santa Cruz Biotechnology Inc.) (72) antibodies were added and reacted for 6 hours at $4^{\circ} \mathrm{C}$. These immunocomplexes were incubated on protein $\mathrm{A} / \mathrm{G}$-agarose beads at $4^{\circ} \mathrm{C}$ overnight. After washing with lysis buffer 3 times, the immunocomplexes were examined by Western blot with anti-CXCR4 (Millipore) and anti-PrPC (M-20; Santa Cruz Biotechnology Inc.) antibodies.

Animal brain ischemia/reperfusion model. Adult male Sprague-Dawley rats were used in this study. Animals were subjected to 3-vessel ligation, as previously described (51). All surgical procedures were performed using sterile/aseptic techniques in accordance with institutional guidelines. All animal experiments were approved by the Institutional Review Board of Animal Experiments, China Medical University Hospital.

Intracerebral transplantation of hOECs/ONFs. For cell labeling, cells were cultured in DMEM (Sigma-Aldrich) with $10 \% \mathrm{FCS}$ at $37^{\circ} \mathrm{C}$ in a humidified atmosphere of $5 \% \mathrm{CO}_{2} / 95 \%$ air and antibiotics. Prior to transplantation, the cells were incubated with $1 \mu \mathrm{g} / \mathrm{ml}$ bis-benzimide (labels nuclei with blue fluorescence) (Hoechst 33342; Sigma-Aldrich) for 5 hours at $37^{\circ} \mathrm{C}$. The labeled cells were then collected and washed in PBS 3 times. Nucleated hOECs/ONFs were counted using a cytometer to ensure an adequate cell number for transplantation. One day after brain ischemia, the adult male
Sprague-Dawley rats (weight, >300 g; age, 7-8 weeks) were anesthetized with chloral hydrate $(0.4 \mathrm{~g} / \mathrm{kg}$, i.p.). Then they were injected stereotaxically with approximately $1 \times 10^{6}$ cells in 3-5 $\mu$ l DMEM medium through a 26-gauge Hamilton syringe into 3 cortical areas adjacent to the right MCA, 3.0-5.0 $\mathrm{mm}$ below the dura as previously described (51). Cyclosporin A (CsA; $1 \mathrm{mg} / \mathrm{kg} / \mathrm{d}$, i.p.; Novartis) injections were given daily to each experimental rat from the day after cerebral ischemia for 3 weeks. An equal volume of CsA was injected to the transplantation group and saline control group, as previously described (73).

Neurological behavioral measurement. Behavioral assessments were performed 5 days before cerebral ischemia and 1, 7, 14, and 28 days after cell transplantation. The tests measured body asymmetry, locomotor activity, and grip strength $(51,74)$. The baseline scores were recorded in order to normalize those taken after cerebral ischemia, as previously described. Grip strength was analyzed using a Grip Strength Meter (TSE Systems) as previously described, with modification (74). In brief, the grip strength ratio for each forelimb was measured separately and was calculated as the ratio of the mean strength $(n=20$ pulls) of the side contralateral to the ischemia to that of the ipsilateral side. In addition, the ratio of grip strength after treatment to that before treatment was calculated; the changes are presented relative to the pretreatment value.

FDG-PET examination. Since glucose metabolism is strongly correlated with functional plasticity of the brain, experimental rats were examined using microPET scanning of FDG to measure relative glucose metabolic activity, as previously described (75). In brief, ${ }^{18} \mathrm{~F}$ was produced by the ${ }^{18} \mathrm{O}(\mathrm{p}, \mathrm{n}){ }^{18} \mathrm{~F}$ nuclear reaction in a cyclotron at China Medical University and Hospital, and FDG was synthesized as previously described (76) with an automated FDG synthesis system (Nihonkokan). Data were collected with a high-resolution small-animal PET (microPET Rodent R4; Concorde Microsystems). The system parameters were described by Carmichael et al. (77). After 4 weeks of each treatment, animals were anesthetized with chloral hydrate $(0.4 \mathrm{~g} / \mathrm{kg}$, i.p.), and the head was fixed in a customized stereotactic head holder and positioned in the microPET scanner. Then the animals were given an intravenous bolus injection of FDG $(200-250 \mu \mathrm{Ci} / \mathrm{rat})$ dissolved in $0.5 \mathrm{ml}$ saline. Data acquisition began at the same time and continued for 60 minutes in one bed position using a 3D acquisition protocol. The image data acquired from microPET were displayed and analyzed by IDL version 5.5 (Research Systems) and ASIPro version 3.2 (Concorde Microsystems) software. FDGPET images were reconstructed using a posterior-based 3D iterative algorithm (78) and overlaid on MR templates to confirm anatomical location (79). Coronal sections for striatal and cortical measurements represented brain areas between 0 and $+1 \mathrm{~mm}$ from the bregma, while thalamic measurements were between -2 and $-3 \mathrm{~mm}$ from the bregma, as estimated by visual inspection of the contralateral side. The relative metabolic activity in regions of interest of the striatum and cortex was expressed as percent deficit as previously described with modification (77).

BrdU labeling and BrdU IHC. BrdU (Sigma-Aldrich), a thymidine analog that is incorporated into the DNA of dividing cells during $S$ phase, was used for mitotic labeling by a protocol described previously (80). Briefly, a pulse-labeling method was used to observe the time course of proliferative cells in the brain after cerebral ischemia. Experimental rats were i.p. injected with BrdU $(50 \mathrm{mg} / \mathrm{kg})$ every 4 hours for 12 hours before sacrifice. A cumulative labeling method was used to examine the population of proliferative cells during 14 days of cerebral ischemia. Rats received daily injections of $\mathrm{BrdU}(50 \mathrm{mg} / \mathrm{kg}$, i.p.) for 14 consecutive days, starting the day after MCA ligation. These rats were sacrificed 14 days after the last injection. The BrdU immunostaining procedure with a specific antibody against BrdU (1:400; Boehringer Mannheim) and quantification of BrdU immunoreactive cells have been described previously (80). In brief, experimental rats' brains were fixed by transcardial perfusion with saline, fol- 
lowed by perfusion and immersion in $4 \%$ paraformaldehyde. Subsequently, the brain samples were dehydrated in $30 \%$ sucrose. After brains were frozen on dry ice, a series of adjacent 6 - $\mu \mathrm{m}$-thick sections were cut in the coronal plane with a cryostat, stained with $\mathrm{H} \& \mathrm{E}$, and observed by light microscopy (E600; Nikon). For BrdU immunostaining, DNA was first denatured by incubating each section in $50 \%$ formamide in $2 \times$ standard saline citrate at $65^{\circ} \mathrm{C}$ for 2 hours, then in $2 \mathrm{~N} \mathrm{HCl}$ at $37^{\circ} \mathrm{C}$ for 30 minutes, and finally rinsed in $0.1 \mathrm{M}$ boric acid with $\mathrm{pH}$ 8.5. Sections were then rinsed with Tris buffer and treated with $1 \% \mathrm{H}_{2} \mathrm{O}_{2}$ to block endogenous peroxidase. The immunostaining procedure was performed using the labeled streptavidinbiotin (LSAB) method (LSAB-2 Kit, Peroxidase; Dako). Tissue slides were incubated with the appropriate diluted antibodies against BrdU (for nuclear identification; 1:400; Boehringer Mannheim) at room temperature for 1 hour. After washing with Tris-buffered saline containing $0.1 \%$ Tween-20, the specimens were sequentially incubated for 10-30 minutes with biotinylated anti-rabbit and anti-mouse (1:200; R\&D Systems) immunoglobulins and peroxidase-labeled streptavidin.

Preparation of transgenic GFP-chimeric mice. In order to verify the enhancement of the BMSC mobilization and homing into brain following hOEC/ ONF implantation, transgenic GFP-chimeric mice generated as previously reported were used (81). In brief, both ends of the femur and tibia were penetrated using a syringe with a 25 -gauge needle, and the marrow was flushed out with sterile saline. Total marrow from 1 femur was diluted to $1 \mathrm{ml}$, then strained through $30-\mu \mathrm{m}$ Spectramesh (Fisher Scientific). Before bone marrow transplantation, female recipient wild-type (C57BL/6 mice) underwent whole-body gamma irradiation with ${ }^{137} \mathrm{Cs}$ using a Gammacell 40 irradiator (MDS Nordion). A total dose of 9 Gy was administered to ablate the whole bone marrow. The mice received rescuing bone marrow transplantations within 24 hours of irradiation. Donor bone marrow was injected into the recipient animal's tail as an $80-\mu \mathrm{l}$ cell suspension containing $3 \times 10^{6}$ cells. At 3 weeks after transplantation, mice were anesthetized with chloral hydrate $(0.3 \mathrm{~g} / \mathrm{kg}$, i.p.) and subjected to right MCA ligation and bilateral common carotid artery clamping for 60 minutes, as previously described with modification (82). Then, 60 minutes after arterial ligation, experimental mice were implanted stereotactically with hOECs/ ONFs $\left(2 \times 10^{5}\right.$ cells) or vehicle ( $200 \mu \mathrm{l}$ saline) through a 30 -gauge Hamilton syringe into 2 cortical areas, $2.0-3.0 \mathrm{~mm}$ below the dura. The approximate coordinates for these sites were $0.5-1.0 \mathrm{~mm}$ anterior to the bregma, $1.5-2.0$ $\mathrm{mm}$ lateral to the midline, $1-1.5 \mathrm{~mm}$ posterior to the bregma, and 2-2.5 $\mathrm{mm}$ lateral to the midline.

Western blot analysis for expression of antiapoptotic proteins and ELISA for growth factor in vivo. Experimental rats were anesthetized with chloral hydrate $(0.4 \mathrm{~g} / \mathrm{kg}$, i.p.) $3,7,14$, and 28 days after initiation of the treatments. Ischemic cortical and striatal areas were evacuated on ice immediately. Subsequently, these brain tissue samples were homogenized with a plastic homogenizer, and growth factors were measured by ELISA analysis, as previously described with modification (83), with a Quantikine ELISA kit (R\&D Systems) following the manufacturer's instructions. Optical density was measured using a spectrophotometer (Molecular Devices). The standard curves were obtained using the program SoftMax (Molecular Devices). Western blot analysis was conducted with specific antibodies against $\mathrm{Bcl}-2$ (1:200; Santa Cruz Biotechnology Inc.), Bcl-xL (1:200; BD Biosciences), Bad (1:200; Santa Cruz Biotechnology Inc.), Bax (1:200; BD Biosciences), and actin (1:2,000; Santa Cruz Biotechnology Inc.).

Immunohistochemical assessment of brain tissue. Animals were anesthetized with chloral hydrate $(0.4 \mathrm{~g} / \mathrm{kg}$, i.p.) and their brains fixed by transcardial perfusion with saline, followed by perfusion with and immersion in $4 \%$ paraformaldehyde as previously described (51).

FISH analysis. To demonstrate the xenografted human cells in the rats' brain, FISH analysis was performed as previously described (63). In brief, cryostat sections $6 \mu \mathrm{m}$ in thickness were fixed 3 times, for 10 minutes each, in Carnoy fixative. Tissue samples were digested with proteinase $\mathrm{K}$ $\left(10 \mu \mathrm{g} / \mathrm{ml}\right.$; Sigma-Aldrich) for 5 minutes at $37^{\circ} \mathrm{C}$, washed with water, and then rinsed in $2 \times$ SSC for 3 minutes and air dried again. Sections were denatured at $85^{\circ} \mathrm{C}$ for 2 minutes in preheated $70 \%$ formamide and $2 \times$ SSC buffer, $\mathrm{pH} 7.0$, and were then quenched with ice-cold $70 \%$ ethanol for 1.5 minutes. The human X chromosome probe labeled with Cy 3 (Star*FISH; Cambio) was heated to $72^{\circ} \mathrm{C}$ for 5 minutes, placed in a $37^{\circ} \mathrm{C}$ water bath for 3 hours to preanneal, and then applied to the sections at $45^{\circ} \mathrm{C}$. Coverslips were placed on the sections for incubation overnight in a hydrated slide box at $42^{\circ} \mathrm{C}$. The next day, the coverslips were removed in preheated $2 \times \mathrm{SSC}$ buffer, $\mathrm{pH} 7.0$, at $45^{\circ} \mathrm{C}$. The sections were washed twice in preheated $50 \%$ formamide in $2 \times \mathrm{SSC}$ buffer for 5 minutes each at $45^{\circ} \mathrm{C}$ and were then gently washed twice in preheated $0.1 \times$ SSC buffer for 5 minutes each at $45^{\circ} \mathrm{C}$. Multicolored fluorescence staining of tissues was analyzed by a laser scanning confocal microscope (LSM510; Zeiss). Counting of FISH signal-positive nuclei was accomplished by systematically examining the FISH-stained tissue, field by field, under $\times 630$ magnification.

Laser scanning confocal microscopy for immunofluorescence colocalization analysis. In order to identify whether cell type-specific markers colocalized with endogenous stem cells in the ischemic rat brain, we labeled endogenous stem cells with BrdU, and exogenous cells (transplanted hOECs/ONFs) were labeled with bis-benzimide (blue fluorescence). Immunofluorescence colocalization analysis was performed with laser scanning confocal microscopy. In immunofluorescence colocalization of hOEC/ONF cells and cell-specific marker studies, each coronal section was treated with cell-specific antibodies: $\mathrm{vWF}$ (for endothelial cells; 1:200; Dako), GFAP (for astrocytes; 1:300), Neu-N (for neuronal nuclei; 1:50), nestin (for neuronal dendrites; $1: 50$ ), and MAP-2 (for neuronal dendrites; 1:300), all from Millipore; SDF-1 $\alpha$ (1:200) and CXCR4 (1:200) from Torrey Pines Biolabs; and PrPC (1:300; M20; Santa Cruz Biotechnology Inc.). The secondary antibodies in the subsequent visualization reaction were Alexa Fluor-conjugated donkey anti-mouse 555, anti-rabbit 555, or anti-rat 555 (1:500; Molecular Probes; Invitrogen) (51).

Assessment of neurite regeneration and Western blotting of $P r P^{C}$ and $C X C R 4$ expression in vivo. Brain tissue samples were immunostained to measure neurite outgrowth. Measurement of neurite regeneration was performed as described previously (69). Briefly, brain tissue samples from each experimental rat were fixed and immunostained with specific antibody against $\beta$-tubulin (1:400; Sigma-Aldrich). For quantification analysis, neurons with processes greater than twice the cell body diameter were counted as neurite-bearing cells. The length of the longest neurite of each neuron was measured from digitized images and quantified using imaging analysis software (SigmaScan 4.01.003). Analysis of the expression of $\operatorname{Pr} \mathrm{PC}^{\mathrm{C}}$ and CXCR4 was performed with specific antibody of $\operatorname{PrPC}(1: 300 ;$ M20; Santa Cruz Biotechnology Inc.) and CXCR4 (1:300; Millipore) in a Western blot as described above. $\operatorname{PrPC}^{\mathrm{C}}$ and CXCR4 activation was inhibited with PrPC-blocking antibody (10 $\mu \mathrm{g} / \mathrm{ml} ; 6 \mathrm{H} 4$; Prionics), CXCR4 neutralizing antibody (R\&D Systems), and control human IgG (Sigma-Aldrich). The blocking protocol to inhibit $\operatorname{PrP}^{\mathrm{C}}$ activation involved pretreatment of the hOECs/ONFs $\left(2 \times 10^{5}\right.$ cells $)$ with anti-PrPC blocking antibody for 24 hours as described previously with modification (84). In addition, the CXCR4 was neutralized by i.p. injection of CXCR4 neutralizing antibody $(1 \mathrm{mg} /$ rat) twice weekly for 2 weeks as described previously (85). Expression of $\mathrm{PrP}^{\mathrm{C}}$ and CXCR4, assay of neurite outgrowth, and neurological behavioral measurement (described above) were used to evaluate the outcome of the 4 treatment protocols (hOECs/ONFs; hOECs/ONFs with PrPC-blocking antibody; hOECs/ONFs with CXCR4-blocking antibody; and hOECs/ ONFs with control human IgG).

Generation of $\mathrm{PrP}^{\mathrm{C}}$-knockout mice. The $\mathrm{PrP}^{+/+}$mice used in this study were wild-type $\mathrm{C} 57 \mathrm{BL} / 6$ mice. $\mathrm{PrP}^{\mathrm{C}}$-knockout $(\mathrm{PrPo} / 0)$ mice were a kind gift from 
Charles Weissmann, Institute of Neurology, London, United Kingdom, as previously described (86). Neurite regeneration after stroke was evaluated in the $\mathrm{PrP}^{+/+}$and $\mathrm{Pr} \mathrm{P}^{\circ / 0}$ mice after hOEC/ONF $\left(1 \times 10^{5}\right.$ cells $)$ implantation as mentioned above.

Statistics. All observers in this study were blinded to the actual conditions of the experiment to minimize observer bias. Results are expressed as mean \pm SEM. The behavioral scores were evaluated for normality, and for normally distributed data, 2-tailed Student's $t$ tests were used to evaluate mean differences between the control and the treated groups. Data lacking normal distribution were analyzed by 1 -way ANOVA. A value of $P<0.05$ was taken as significant.

\section{Acknowledgments}

We would like to thank Harry Wilson and M. Loney of Academia Sinica for their critical reading of the manuscript. This work was supported in part by research grants from the Chen-Han Founda- tion for Education, Academia Sinica (94M003), the Health Research Institute (Republic of China) (NHRI-CN-SC9303S), and the National Science Council (Republic of China) (NSC95-2314-B-303-003).

Received for publication October 30, 2007, and accepted in revised form April 16, 2008.

Address correspondence to: Hung Li, Institute of Molecular Biology, Academia Sinica, 128 Sec. 2, Academia Road, Nanking, Taipei 11529, Republic of China. Phone: 886-2-2788-0460; Fax: 886-2-2782-6085; E-mail: hungli@ccvax.sinica.edu.tw. Or to: Demeral David Liu, Department of Dentistry, China Medical University Hospital, 2 Yuh-Der Rd, Taichung 40447, Republic of China. Phone: 886-4-22052121 ext. 6034; Fax: 886-4-22080666; E-mail: liudm@mail.ndmctsgh.edu.tw.
1. Li, Y., Decherchi, P., and Raisman, G. 2003. Transplantation of olfactory ensheathing cells into spinal cord lesions restores breathing and climbing. J. Neurosci. 23:727-731.

2. Field, P., Li, Y., and Raisman, G. 2003. Ensheathment of the olfactory nerves in the adult rat. J. Neurocytol. 32:317-324.

3. Kato, T., Honmou, O., Uede, T., Hashi, K., and Kocsis, J.D. 2000. Transplantation of human olfactory ensheathing cells elicits remyelination of demyelinated rat spinal cord. Glia. 30:209-218.

4. Agrawal, A.K., et al. 2004. Olfactory ensheathing cell transplantation restores functional deficits in rat model of Parkinson's disease: a cotransplantation approach with fetal ventral mesencephalic cells. Neurobiol. Dis. 16:516-526.

5. Devon, R., and Doucette, R. 1992. Olfactory ensheathing cells myelinate dorsal root ganglion neurites. Brain Res. 589:175-179.

6. Imaizumi, T., Lankford, K.L., Waxman, S.G., Greer, C.A., and Kocsis, J.D. 1998. Transplanted olfactory ensheathing cells remyelinate and enhance axonal conduction in the demyelinated dorsal columns of the rat spinal cord. J. Neurosci. 18:6176-6185.

7. Deumens, R., et al. 2006. Olfactory ensheathing cells, olfactory nerve fibroblasts and biomatrices to promote long-distance axon regrowth and functional recovery in the dorsally hemisected adult rat spinal cord. Exp. Neurol. 200:89-103.

8. Deumens, R., et al. 2006. Chronically injured corticospinal axons do not cross large spinal lesion gaps after a multifactorial transplantation strategy using olfactory ensheathing cell/olfactory nerve fibroblast-biomatrix bridges. J. Neurosci. Res. 83:811-820.

9. Jones, L.L., Oudega, M., Bunge, M.B., and Tuszynski, M.H. 2001. Neurotrophic factors, cellular bridges and gene therapy for spinal cord injury. J. Physiol. 533:83-89.

10. Chen, J., et al. 2005. Atorvastatin induction of VEGF and BDNF promotes brain plasticity after stroke in mice. J. Cereb. Blood Flow. Metab. 25:281-290.

11. Lopez-Vales, R., Garcia-Alias, G., Fores, J., Navarro, X., and Verdu, E. 2004. Increased expression of cyclo-oxygenase 2 and vascular endothelial growth factor in lesioned spinal cord by transplanted olfactory ensheathing cells. J. Neurotrauma. 21:1031-1043.

12. Kurozumi, K., et al. 2005. Mesenchymal stem cells that produce neurotrophic factors reduce ischemic damage in the rat middle cerebral artery occlusion model. Mol. Ther. 11:96-104.

13. Hill, W.D., et al. 2004. SDF-1 (CXCL12) is upregulated in the ischemic penumbra following stroke: association with bone marrow cell homing to injury. J. Neuropathol. Exp. Neurol. 63:84-96.

14. Zheng, J., et al. 1999. Intracellular CXCR4 signaling, neuronal apoptosis and neuropathogenic mechanisms of HIV-1-associated dementia. J. Neuroimmunol. 98:185-200.

15. Evert, B.O., et al. 2001. Inflammatory genes are upregulated in expanded ataxin-3-expressing cell lines and spinocerebellar ataxia type 3 brains. J. Neurosci. 21:5389-5396.

16. Jo, D.Y., Rafii, S., Hamada, T., and Moore, M.A. 2000. Chemotaxis of primitive hematopoietic cells in response to stromal cell-derived factor-1. J. Clin. Invest. 105:101-111.

17. Lapidot, T., Dar, A., and Kollet, O. 2005. How do stem cells find their way home? Blood. 106:1901-1910.

18. Shirozu, M., et al. 1995. Structure and chromosomal localization of the human stromal cell-derived factor 1 (SDF1) gene. Genomics. 28:495-500.

19. Bueler, H., et al. 1993. Mice devoid of PrP are resistant to scrapie. Cell. 73:1339-1347.

20. Roucou, X., Gains, M., and LeBlanc, A.C. 2004. Neuroprotective functions of prion protein. J. Neurosci. Res. 75:153-161.

21. Sales, N., et al. 1998. Cellular prion protein localization in rodent and primate brain. Eur. J. Neurosci. 10:2464-2471.

22. Sales, N., et al. 2002. Developmental expression of the cellular prion protein in elongating axons. Eur. J. Neurosci. 15:1163-1177.

23. Graner, E., et al. 2000. Cellular prion protein binds laminin and mediates neuritogenesis. Brain Res. Mol. Brain Res. 76:85-92.

24. Graner, E., et al. 2000. Laminin-induced PC-12 cell differentiation is inhibited following laser inactivation of cellular prion protein. FEBS Lett. 482:257-260

25. Raisman, G. 2001. Olfactory ensheathing cells - another miracle cure for spinal cord injury? Nat. Rev. Neurosci. 2:369-375.

26. Cao, L., et al. 2004. Olfactory ensheathing cells genetically modified to secrete GDNF to promote spinal cord repair. Brain. 127:535-549.

27. Lakatos, A., Smith, P.M., Barnett, S.C., and Franklin, R.J. 2003. Meningeal cells enhance limited CNS remyelination by transplanted olfactory ensheathing cells. Brain. 126:598-609.

28. Bang, A.G., and Goulding, M.D. 1996. Regulation of vertebrate neural cell fate by transcription factors. Curr. Opin. Neurobiol. 6:25-32.

29. Temple, S., and Alvarez-Buylla, A. 1999. Stem cells in the adult mammalian central nervous system. Curr. Opin. Neurobiol. 9:135-141.

30. Schwob, J.E. 2002. Neural regeneration and the peripheral olfactory system. Anat. Rec. 269:33-49.

31. Jang, W., Youngentob, S.L., and Schwob, J.E. 2003. Globose basal cells are required for reconstitution of olfactory epithelium after methyl bromide lesion. J. Comp. Neurol. 460:123-140.

32. Ma, Q., Sommer, L., Cserjesi, P., and Anderson, D.J. 1997. Mash1 and neurogenin 1 expression patterns define complementary domains of neuroepithe- lium in the developing CNS and are correlated with regions expressing notch ligands. J. Neurosci. 17:3644-3652.

33. Guillemot, F. 1999. Vertebrate bHLH genes and the determination of neuronal fates. Exp. Cell Res. 253:357-364.

34. Mackay-Sima, A., and Chuahb, M.I. 2000. Neurotrophic factors in the primary olfactory pathway. Prog. Neurobiol. 62:527-559.

35. Buckland, M.E., and Cunningham, A.M. 1999. Alterations in expression of the neurotrophic factors glial cell line-derived neurotrophic factor, ciliary neurotrophic factor and brain-derived neurotrophic factor, in the target-deprived olfactory neuroepithelium. Neuroscience. 90:333-347.

36. Saarma, M., and Sariola, H. 1999. Other neurotrophic factors: glial cell line-derived neurotrophic factor (GDNF). Microsc. Res. Tech. 45:292-302.

37. Thewke, D.P., and Seeds, N.W. 1996. Expression of hepatocyte growth factor/scatter factor, its receptor, c-met, and tissue-type plasminogen activator during development of the murine olfactory system. J. Neurosci. 16:6933-6944.

38. Zhang, S.C., and Fedoroff, S. 1997. Cellular localization of stem cell factor and c-kit receptor in the mouse nervous system. J. Neurosci. Res. 47:1-15.

39. Zhang, K., et al. 2003. HIV-induced metalloproteinase processing of the chemokine stromal cell derived factor-1 causes neurodegeneration. Nat. Neurosci. 6:1064-1071.

40. Barber, P.C., and Lindsay, R.M. 1982. Schwann cells of the olfactory nerves contain glial fibrillary acidic protein and resemble astrocytes. Neuroscience. 7:3077-3090.

41. Hill, W.D., et al. 2004. SDF-1 (CXCL12) is upregulated in the ischemic penumbra following stroke: association with bone marrow cell homing to injury. J. Neuropathol. Exp. Neurol. 63:84-96.

42. Arakawa, Y., et al. 2003. Control of axon elongation via an SDF-1alpha/Rho/mDia pathway in cultured cerebellar granule neurons. J. Cell Biol. 161:381-391.

43. Beites, C.L., Kawauchi, S., Crocker, C.E., and Calof, A.L. 2005. Identification and molecular regulation of neural stem cells in the olfactory epithelium. Exp. Cell Res. 306:309-316.

44. Imaizumi, T., Lankford, K.L., and Kocsis, J.D. 2000. Transplantation of olfactory ensheathing cells or Schwann cells restores rapid and secure conduction across the transected spinal cord. Brain Res. 854:70-78.

45. Li, Y., Field, P.M., and Raisman, G. 1997. Repair of adult rat corticospinal tract by transplants of olfactory ensheathing cells. Science. 277:2000-2002.

46. Ramon-Cueto, A., Plant, G.W., Avila, J., and Bunge, M.B. 1998. Long-distance axonal regeneration in the transected adult rat spinal cord is promoted by olfactory ensheathing glia transplants. J. Neurosci. 
18:3803-3815

47. Ramon-Cueto, A., Cordero, M.I., Santos-Benito, F.F., and Avila, J. 2000. Functional recovery of paraplegic rats and motor axon regeneration in their spinal cords by olfactory ensheathing glia. Neuron. 25:425-435.

48. Deumens, R., et al. 2006. Neurite outgrowth promoting effects of enriched and mixed OEC/ONF cultures. Neurosci. Lett. 397:20-24.

49. Peled, A., et al. 1999. The chemokine SDF-1 stimulates integrin-mediated arrest of CD34(+) cells on vascular endothelium under shear flow. J. Clin. Invest. 104:1199-1211.

50. Stumm, R.K., et al. 2002. A dual role for the SDF-1/ CXCR4 chemokine receptor system in adult brain: isoform-selective regulation of SDF-1 expression modulates CXCR4-dependent neuronal plasticity and cerebral leukocyte recruitment after focal ischemia. J. Neurosci. 22:5865-5878.

51. Shyu, W.C., et al. 2004. Functional recovery of stroke rats induced by granulocyte colony-stimulating factor-stimulated stem cells. Circulation. 110:1847-1854.

52. Woodhall, E., West, A.K., and Chuah, M.I. 2001. Cultured olfactory ensheathing cells express nerve growth factor, brain-derived neurotrophic factor, glia cell line-derived neurotrophic factor and their receptors. Brain Res. Mol. Brain Res. 88:203-213.

53. Ramon-Cueto, A., and Nieto-Sampedro, M. 1992. Glial cells from adult rat olfactory bulb: immunocytochemical properties of pure cultures of ensheathing cells. Neuroscience. 47:213-220.

54. Sonigra, R.J., Brighton, P.C., Jacoby, J., Hall, S., and Wigley, C.B. 1999. Adult rat olfactory nerve ensheathing cells are effective promoters of adult central nervous system neurite outgrowth in coculture. Glia. 25:256-269.

55. Schmitt-Ulms, G., et al. 2004. Time-controlled transcardiac perfusion cross-linking for the study of protein interactions in complex tissues. Nat. Biotechnol. 22:724-731.

56. Hundt, C., et al. 2001. Identification of interaction domains of the prion protein with its $37-\mathrm{kDa} / 67$ $\mathrm{kDa}$ laminin receptor. EMBO J. 20:5876-5886.

57. Rieger, R., Edenhofer, F., Lasmezas, C.I., and Weiss, S. 1997. The human $37-\mathrm{kDa}$ laminin receptor precursor interacts with the prion protein in eukaryotic cells. Nat. Med. 3:1383-1388.

58. Pujol, F., Kitabgi, P., and Boudin, H. 2005. The chemokine SDF-1 differentially regulates axonal elongation and branching in hippocampal neurons. J. Cell Sci. 118:1071-1080.

59. Vilz, T.O., et al. 2005. The SDF-1/CXCR4 pathway and the development of the cerebellar system. Eur.
J. Neurosci. 22:1831-1839.

60. Xiang, Y., et al. 2002. Nerve growth cone guidance mediated by G protein-coupled receptors. Nat. Neurosci. 5:843-848.

61. Le, Y., et al. 2002. Receptors for chemotactic formyl peptides as pharmacological targets. Int. Immunopharmacol. 2:1-13.

62. Borrell, V., and Marin, O. 2006. Meninges control tangential migration of hem-derived Cajal-Retzius cells via CXCL12/CXCR4 signaling. Nat. Neurosci. 9:1284-1293.

63. Terada, N., et al. 2002. Bone marrow cells adopt the phenotype of other cells by spontaneous cell fusion. Nature. 416:542-545.

64. Ying, Q.L., Nichols, J., Evans, E.P., and Smith, A.G. 2002. Changing potency by spontaneous fusion. Nature. 416:545-548.

65. Zhang, S., Shpall, E., Willerson, J.T., and Yeh, E.T. 2007. Fusion of human hematopoietic progenitor cells and murine cardiomyocytes is mediated by alpha 4 beta 1 integrin/vascular cell adhesion molecule-1 interaction. Circ. Res. 100:693-702.

66. Prockop, D.J., Gregory, C.A., and Spees, J.L. 2003. One strategy for cell and gene therapy: harnessing the power of adult stem cells to repair tissues. Proc. Natl. Acad. Sci. U. S. A. 100(Suppl. 1):11917-11923.

67. Sasaki, M., Lankford, K.L., Zemedkun, M., and Kocsis, J.D. 2004. Identified olfactory ensheathing cells transplanted into the transected dorsal funiculus bridge the lesion and form myelin. J. Neurosci. 24:8485-8493.

68. Murphy, T.H., Schnaar, R.L., and Coyle, J.T. 1990. Immature cortical neurons are uniquely sensitive to glutamate toxicity by inhibition of cystine uptake. FASEB J. 4:1624-1633.

69. Cafferty, W.B., et al. 2004. Conditioning injuryinduced spinal axon regeneration fails in interleukin-6 knock-out mice. J. Neurosci. 24:4432-4443.

70. Zhang, Y., Lu, H., Dazin, P., and Kapila, Y. 2004 Functional differences between integrin alpha4 and integrins alpha5/alphav in modulating the motility of human oral squamous carcinoma cells in response to the $\mathrm{V}$ region and heparin-binding domain of fibronectin. Exp. Cell Res. 295:48-58.

71. Ding, Y., et al. 2006. The 73-kDa heat shock cognate protein is a CXCR4 binding protein that regulates the receptor endocytosis and the receptor-mediated chemotaxis. Mol. Pharmacol. 69:1269-1279.

72. Mattei, V., et al. 2004. Prion protein is a component of the multimolecular signaling complex involved in T cell activation. FEBS Lett. 560:14-18.

73. Wennersten, A., et al. 2006. Sustained survival of xenografted human neural stem/progenitor cells in experimental brain trauma despite discontinuation of immunosuppression. Exp. Neurol. 199:339-347. 74. Dunnett, S.B., Torres, E.M., and Annett, L.E. 1998. A lateralised grip strength test to evaluate unilateral nigrostriatal lesions in rats. Neurosci. Lett. 246:1-4.

75. Matsumura, A., et al. 2003. Assessment of microPET performance in analyzing the rat brain under different types of anesthesia: comparison between quantitative data obtained with microPET and ex vivo autoradiography. Neuroimage. 20:2040-2050.

76. Hamacher, K., Coenen, H.H., and Stocklin, G. 1986. Efficient stereospecific synthesis of no-carrier-added 2-[18F]-fluoro-2-deoxy-D-glucose using aminopolyether supported nucleophilic substitution. J. Nucl. Med. 27:235-238.

77. Carmichael, S.T., Tatsukawa, K., Katsman, D., Tsuyuguchi, N., and Kornblum, H.I. 2004. Evolution of diaschisis in a focal stroke model. Stroke. 35:758-763.

78. Kornblum, H.I., et al. 2000. In vivo imaging of neuronal activation and plasticity in the rat brain by high resolution positron emission tomography (microPET). Nat. Biotechnol. 18:655-660.

79. Brownell, A.L., Livni, E., Galpern, W., and Isacson, O. 1998. In vivo PET imaging in rat of dopamine terminals reveals functional neural transplants. Ann. Neurol. 43:387-390.

80. Zhang, R.L., Zhang, Z.G., Zhang, L., and Chopp, M. 2001. Proliferation and differentiation of progenitor cells in the cortex and the subventricular zone in the adult rat after focal cerebral ischemia. Neuroscience. 105:33-41.

81. Hess, D.C., et al. 2002. Bone marrow as a source of endothelial cells and NeuN-expressing cells After stroke. Stroke. 33:1362-1368.

82. Chen, S.T., Hsu, C.Y., Hogan, E.L., Maricq, H., and Balentine, J.D. 1986. A model of focal ischemic stroke in the rat: reproducible extensive cortical infarction. Stroke. 17:738-743.

83. Franklin, T.B., and Perrot-Sinal, T.S. 2006. Sex and ovarian steroids modulate brain-derived neurotrophic factor (BDNF) protein levels in rat hippocampus under stressful and non-stressful conditions. Psychoneuroendocrinology. 31:38-48.

84. Lopes, M.H., et al. 2005. Interaction of cellular prion and stress-inducible protein 1 promotes neuritogenesis and neuroprotection by distinct signaling pathways. J. Neurosci. 25:11330-11339.

85. Muller, A., et al. 2001. Involvement of chemokine receptors in breast cancer metastasis. Nature. 410:50-56.

86. Rossi, D., et al. 2001. Onset of ataxia and Purkinje cell loss in PrP null mice inversely correlated with $\mathrm{Dpl}$ level in brain. EMBO J. 20:694-702. 